\title{
Regularity in Sobolev and Besov Spaces for Parabolic Problems on Domains of Polyhedral Type
}

\author{
Stephan Dahlke ${ }^{1} \cdot$ Cornelia Schneider ${ }^{2}$
}

Received: 26 October 2020 / Accepted: 12 May 2021 / Published online: 4 June 2021

(c) The Author(s) 2021

\begin{abstract}
This paper is concerned with the regularity of solutions to linear and nonlinear evolution equations extending our findings in Dahlke and Schneider (Anal Appl 17(2):235-291, 2019, Thms. 4.5, 4.9, 4.12, 4.14) to domains of polyhedral type. In particular, we study the smoothness in the specific scale $B_{\tau, \tau}^{r}, \frac{1}{\tau}=\frac{r}{d}+\frac{1}{p}$ of Besov spaces. The regularity in these spaces determines the approximation order that can be achieved by adaptive and other nonlinear approximation schemes. We show that for all cases under consideration the Besov regularity is high enough to justify the use of adaptive algorithms.
\end{abstract}

Keywords Parabolic evolution equations · Besov spaces · Kondratiev spaces · Adaptive algorithms

Mathematics Subject Classification Primary 35B65, 35K55, 46E35 - Secondary 35L15, 35A02, 35K05, 65M12

\section{Introduction}

This paper is concerned with regularity estimates of the solutions to evolution equations in nonsmooth domains of polyhedral type $D \subset \mathbb{R}^{3}$, cf. Definition 1 . In particular, we

The work of Cornelia Schneider has been supported by Deutsche Forschungsgemeinschaft (DFG), Grant No. SCHN 1509/1-2.

$凶$ Cornelia Schneider

cornelia.schneider@math.fau.de

Stephan Dahlke

dahlke@mathematik.uni-marburg.de

1 FB12 Mathematics and Computer Science, Philipps-University Marburg, Hans-Meerwein Straße, Lahnberge, 35032 Marburg, Germany

2 Applied Mathematics III, Friedrich-Alexander University Erlangen-Nuremberg, Cauerstr. 11, 91058 Erlangen, Germany 
study linear $(\varepsilon=0)$ and nonlinear $(\varepsilon>0)$ equations of the form

$$
\frac{\partial}{\partial t} u+(-1)^{m} L\left(t, x, D_{x}\right) u+\varepsilon u^{M}=f \quad \text { in }[0, T] \times D,
$$

with zero initial and Dirichlet boundary conditions, where $m, M \in \mathbb{N}$, and $L$ denotes a uniformly elliptic operator of order $2 m$ with sufficiently smooth coefficients. Special attention is paid to the spatial regularity of the solutions to (1.1) in specific nonstandard smoothness spaces, i.e., in the so-called adaptivity scale of Besov spaces

$$
B_{\tau, \tau}^{r}(D), \quad \frac{1}{\tau}=\frac{r}{3}+\frac{1}{p}, \quad r>0 .
$$

Our investigations are motivated by fundamental questions arising in the context of the numerical treatment of Eq. (1.1). In particular, we aim at justifying the use of adaptive numerical methods for parabolic PDEs. Let us explain these relationships in more detail: In an adaptive strategy, the choice of the underlying degrees of freedom is not a priori fixed but depends on the shape of the unknown solution. In particular, additional degrees of freedom are only spent in regions where the numerical approximation is still 'far away' from the exact solution. Although the basic idea is convincing, adaptive algorithms are hard to implement, so that beforehand a rigorous mathematical analysis to justify their use is highly desirable.

Given an adaptive algorithm based on a dictionary for the solution spaces of the PDE, the best one can expect is an optimal performance in the sense that it realizes the convergence rate of best $N$-term approximation schemes, which serves as a benchmark in this context. Given a dictionary $\Psi=\left\{\psi_{\lambda}\right\}_{\lambda \in \Lambda}$ of functions in a Banach space $X$, the error of best $N$-term approximation is defined as

$$
\sigma_{N}(u ; X)=\inf _{\Gamma \subset \Lambda: \# \Gamma \leq N} \inf _{c_{\lambda}}\left\|u-\sum_{\lambda \in \Gamma} c_{\lambda} \psi_{\lambda} \mid X\right\|,
$$

i.e., as the name suggests we consider the best approximation by linear combinations of the basic functions consisting of at most $N$ terms. In particular, [23, Thm. 11, p. 586] implies for $\tau<p$,

$$
\sigma_{N}\left(u ; L_{p}(D)\right) \leq C N^{-s / d}\left\|u \mid B_{\tau, \tau}^{s}(D)\right\|, \quad \frac{1}{\tau}<\frac{s}{3}+\frac{1}{p} .
$$

Quite recently, it has turned out that the same interrelations also hold for the very important and widespread adaptive finite element schemes. In particular, [27, Thm. 2.2] gives direct estimates,

$$
\sigma_{N}^{F E}\left(u ; L_{p}(D)\right) \leq C N^{-s / d}\left\|u \mid B_{\tau, \tau}^{s}(D)\right\|,
$$

where $\sigma_{N}^{F E}$ denotes the counterpart to the quantity $\sigma_{N}(u ; X)$, which corresponds to wavelet approximations. It can be seen that the achievable order of adaptive algorithms depends on the regularity of the target function in the specific scale of Besov 
spaces (1.2). On the other hand it is the regularity of the solution in the scale of Sobolev spaces, which encodes information on the convergence order for nonadaptive (uniform) methods. From this we can draw the following conclusion: adaptivity is justified, if the Besov regularity of the solution in the Besov scale (1.2) is higher than its Sobolev smoothness!

For the case of elliptic partial differential equations, a lot of positive results in this direction are already established [13-19,30,31]. It is well known that if the domain under consideration, the right-hand side and the coefficients are sufficiently smooth, then the problem is completely regular [1], and there is no reason why the Besov smoothness should be higher than the Sobolev regularity. However, on general Lipschitz domains and in particular in polyhedral domains, the situation changes dramatically. On these domains, singularities at the boundary may occur that diminish the Sobolev regularity of the solution significantly [10,12,28,29,32]. However, the analysis in the above mentioned papers shows that these boundary singularities do not influence the Besov regularity too much, so that the use of adaptive algorithms for elliptic PDEs is completely justified!

In this paper, we study similar questions for evolution equations of the form (1.1) and of associated semilinear versions. To the best of our knowledge, not so many results in this direction are available so far. For parabolic equations, first results for the special case of the heat equation have been reported in [2-4], but for a slightly different scale of Besov spaces.

Our results show in the linear case $\varepsilon=0$ that if the right-hand side as well as its time derivatives are contained in specific Kondratiev spaces, then, for every $t \in[0, T]$ the spatial Besov smoothness of the solution to (1.1) is always larger than $2 m$, provided that some technical conditions on the operator pencils are satisfied, see Theorems 8 and 9 . The reader should observe that the results are independent of the shape of the polyhedral domain, and that the classical Sobolev smoothness is usually limited by $m$, see [35]. Therefore, for every $t$, the spatial Besov regularity is more than twice as high as the Sobolev smoothness, which of course justifies the use of (spatial) adaptive algorithms. Moreover, for smooth domains and right-hand sides in $L_{2}$, the best one could expect would be smoothness order $2 m$ in the classical Sobolev scale. So, the Besov smoothness on polyhedral type domains is at least as high as the Sobolev smoothness on smooth domains.

Afterwards, we generalize this result to nonlinear parabolic equations of the form (1.1). We show that in a sufficiently small ball containing the solution of the corresponding linear equation, there exists a unique solution to (1.1) possessing the same Besov smoothness in the scale (1.2). The proof is performed by a technically quite involved application of the Banach fixed point theorem. The final result is stated in Theorem 10.

The next natural step is to also study the regularity in time direction. For the linear parabolic problem (1.1) with $\varepsilon=0$ we show that the mapping $t \mapsto u(t, \cdot)$ is in fact a $C^{l}$-map into the adaptivity scale of Besov spaces, precisely,

$$
u \in \mathcal{C}^{l, \frac{1}{2}}\left((0, T), B_{\tau, \infty}^{\alpha}(D)\right),
$$


see Theorem 11.

In conclusion, the results presented in this paper imply that for each $t \in(0, T)$ the spatial Besov regularity of the unknown solutions of the problems studied here is much higher than the Sobolev regularity, which justifies the use of spatial adaptive algorithms. This corresponds to the classical time-marching schemes such as the Rothe method. We refer, e.g., to the monographs $[34,42]$ for a detailed discussion. Of course, it would be tempting to employ adaptive strategies in the whole space-time cylinder. First results in this direction have been reported in [41]. To justify also these schemes, Besov regularity in the whole space-time cylinder has to be established. This case will be studied in a forthcoming paper.

Throughout the paper we use the same notation as in [22], which for the convenience of the reader is recalled in Appendix 1.

\section{Sobolev and Kondratiev Spaces}

In this section, we briefly collect the basics concerning weighted and unweighted Sobolev spaces needed later on. In particular, we put $H^{m}=W_{2}^{m}$ and denote by $\stackrel{\circ}{H}^{m}$ the closure of test functions in $H^{m}$ and its dual space by $H^{-m}$. Moreover, $\mathcal{C}^{k, \alpha}, k \in \mathbb{N}_{0}$, stands for the usual Hölder spaces with exponent $\alpha \in(0,1]$. The following generalized version of Sobolev's embedding theorem for Banach-space valued functions will be useful, cf. [40, Thm. 1.2.5].

Theorem 1 (Generalized Sobolev's embedding theorem) Let $1<p<\infty, m \in \mathbb{N}$, $I \subset \mathbb{R}$ be some bounded interval, and $X$ a Banach space. Then

$$
W_{p}^{m}(I, X) \hookrightarrow \mathcal{C}^{m-1,1-\frac{1}{p}}(I, X) .
$$

Here the Banach-valued Sobolev spaces are endowed with the norm

$$
\begin{gathered}
\left\|u\left|W_{p}^{m}(I, X)\left\|^{p}:=\sum_{k=0}^{m}\right\| \partial_{t^{k}} u\right| L_{p}(I, X)\right\|^{p} \quad \text { with }\left\|\partial_{t^{k}} u \mid L_{p}(I, X)\right\|^{p} \\
:=\int_{I}\left\|\partial_{t^{k}} u(t) \mid X\right\|^{p} \mathrm{~d} t
\end{gathered}
$$

whereas for the Hölder spaces we use

$$
\left\|u\left|\mathcal{C}^{k, \alpha}(I, X)\|:=\| u\right| C^{k}(I, X)\right\|+\left|u^{(k)}\right|_{C^{\alpha}(I, X)},
$$

where $\left\|u\left|C^{k}(I, X)\left\|=\sum_{j=0}^{k} \max _{t \in I}\right\| u^{(j)}(t)\right| X\right\|$ and $\left|u^{(k)}\right|_{C^{\alpha}(I, X)}=$ $\sup _{s, t \in I} \frac{\left\|u^{(k)}(t)-u^{(k)}(s) \mid X\right\|}{|t-s|^{\alpha}}$.

$s, t \in I$,

$s \neq t$

We collect some notation for specific Banach-space valued Lebesgue and Sobolev spaces, which will be used when studying the regularity of solutions of parabolic PDEs. 
Let $\Omega_{T}:=[0, T] \times \Omega$. Then we abbreviate

$$
L_{p}\left(\Omega_{T}\right):=L_{p}\left([0, T], L_{p}(\Omega)\right) .
$$

Moreover, we put

$$
H^{m, l *}\left(\Omega_{T}\right):=H^{l-1}\left([0, T], \stackrel{\circ}{H}^{m}(\Omega)\right) \cap H^{l}\left([0, T], H^{-m}(\Omega)\right)
$$

normed by

$$
\left\|u\left|H^{m, l *}\left(\Omega_{T}\right)\|=\| u\right| H^{l-1}\left([0, T], \stackrel{\circ}{H}^{m}(\Omega)\right)\right\|+\left\|u \mid H^{l}\left([0, T], H^{-m}(\Omega)\right)\right\| .
$$

\subsection{Kondratiev Spaces}

In the sequel we work to a great extent with weighted Sobolev spaces, the so-called Kondratiev spaces $\mathcal{K}_{p, a}^{m}(\mathcal{O})$, defined as the collection of all $u \in \mathcal{D}^{\prime}(\mathcal{O})$, which have $m$ generalized derivatives satisfying

$$
\left\|u \mid \mathcal{K}_{p, a}^{m}(\mathcal{O})\right\|:=\left(\sum_{|\alpha| \leq m} \int_{\mathcal{O}}|\varrho(x)|^{p(|\alpha|-a)}\left|D_{x}^{\alpha} u(x)\right|^{p} \mathrm{~d} x\right)^{1 / p}<\infty
$$

where $a \in \mathbb{R}, 1<p<\infty, m \in \mathbb{N}_{0}, \alpha \in \mathbb{N}_{0}^{n}$, and the weight function $\varrho: D \rightarrow[0,1]$ is the smooth distance to the singular set of $\mathcal{O}$, i.e., $\varrho$ is a smooth function and in the vicinity of the singular set $S$ it is equivalent to the distance to that set. Clearly, if $\mathcal{O}$ is a polygon in $\mathbb{R}^{2}$ or a polyhedral domain in $\mathbb{R}^{3}$, then the singular set $S$ consists of the vertices of the polygon or the vertices and edges of the polyhedra, respectively.

It follows directly from (2.2) that the scale of Kondratiev spaces is monotone in $m$ and $a$, i.e.,

$$
\mathcal{K}_{p, a}^{m}(\mathcal{O}) \hookrightarrow \mathcal{K}_{p, a}^{m^{\prime}}(\mathcal{O}) \text { and } \mathcal{K}_{p, a}^{m}(\mathcal{O}) \hookrightarrow \mathcal{K}_{p, a^{\prime}}^{m}(\mathcal{O})
$$

if $m^{\prime}<m$ and $a^{\prime}<a$.

Moreover, generalizing the above concept to functions depending on the time $t \in$ $[0, T]$, we define Kondratiev type spaces, denoted by $L_{q}\left((0, T), \mathcal{K}_{p, a}^{m}(\mathcal{O})\right)$, which contain all functions $u(x, t)$ such that

$$
\begin{aligned}
& \left\|u \mid L_{q}\left((0, T), \mathcal{K}_{p, a}^{m}(\mathcal{O})\right)\right\| \\
& \quad:=\left(\int_{(0, T)}\left(\sum_{|\alpha| \leq m} \int_{\mathcal{O}}|\varrho(x)|^{p(|\alpha|-a)}\left|D_{x}^{\alpha} u(x, t)\right|^{p} \mathrm{~d} x\right)^{q / p} \mathrm{~d} t\right)^{1 / q}<\infty,
\end{aligned}
$$

with $0<q \leq \infty$ and parameters $a, p, m$ as above. 
Fig. 1 Polyhedron

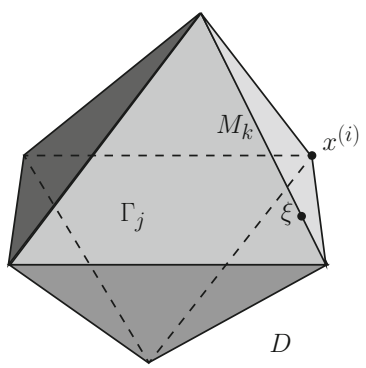

Kondratiev spaces on domains of polyhedral type

For our analysis we make use of several properties of Kondratiev spaces that have been proved in [20]. Therefore, in our later considerations, we will mainly be interested in the case that $\mathcal{O}$ is a bounded domain of polyhedral type.

The precise definition below is taken from Maz'ya and Rossmann [36, Def. 4.1.1] (Fig. 1).

Definition 1 A bounded domain $D \subset \mathbb{R}^{3}$ is defined to be of polyhedral type if the following holds:

(a) The boundary $\partial D$ consists of smooth (of class $C^{\infty}$ ) open two-dimensional manifolds $\Gamma_{j}$ (the faces of $D$ ), $j=1, \ldots, n$, smooth curves $M_{k}$ (the edges), $k=1, \ldots, l$, and vertices $x^{(1)}, \ldots, x^{\left(l^{\prime}\right)}$.

(b) For every $\xi \in M_{k}$ there exists a neighborhood $U_{\xi}$ and a $C^{\infty}$-diffeomorphism $\kappa \xi$ which maps $D \cap U_{\xi}$ onto $\mathcal{D}_{\xi} \cap B_{1}(0)$, where $\mathcal{D}_{\xi} \subset \mathbb{R}^{3}$ is a dihedron, which in polar coordinates can be described as

$$
\mathcal{D}_{\xi}=K \times \mathbb{R}, \quad K=\left\{\left(x_{1}, x_{2}\right): 0<r<\infty,-\theta / 2<\varphi<\theta / 2\right\},
$$

where the opening angle $\theta$ of the 2-dimensional wedge $K$ satisfies $0<\theta \leq 2 \pi$.

(c) For every vertex $x^{(i)}$ there exists a neighborhood $U_{i}$ and a diffeomorphism $\kappa_{i}$ mapping $D \cap U_{i}$ onto $K_{i} \cap B_{1}(0)$, where $K_{i}$ is a polyhedral cone with edges and vertex at the origin.

Remark 1 (i) In the literature many different types of polyhedral domains are considered. A more general version which coincides with the above definition when $d=3$ is discussed in [20]. Further variants of polyhedral domains can be found in Babuška and Guo [7], Bacuta et al. [8], and Mazzucato and Nistor [37].

(ii) Let us point out that 'smooth' domains without edges and/or vertices are admissible in Definition 1. We discuss this further in Sect. 3.2.

Some properties of Kondratiev spaces

Concerning pointwise multiplication the following results are proven in [20].

Corollary 1 (i) Let $m \in \mathbb{N}, a \geq \frac{3}{p}$, and either $1<p<\infty$ and $m>\frac{3}{p}$ or $p=1$ and $m \geq 3$. Then the Kondratiev space $\mathcal{K}_{a, p}^{m}(D)$ is an algebra with respect to 
pointwise multiplication, i.e., there exists a constant c such that

$$
\left\|u v\left|\mathcal{K}_{a, p}^{m}(D)\|\leq c\| u\right| \mathcal{K}_{a, p}^{m}(D)\right\| \cdot\left\|v \mid \mathcal{K}_{a, p}^{m}(D)\right\|
$$

holds for all $u, v \in \mathcal{K}_{a, p}^{m}(D)$.

(ii) Let $\frac{3}{2}<p<\infty, m \in \mathbb{N}$, and $a \geq \frac{3}{p}-1$. Then there exists a constant $c$ such that

$$
\left\|u v\left|\mathcal{K}_{a-1, p}^{m-1}(D)\|\leq c\| u\right| \mathcal{K}_{a+1, p}^{m+1}(D)\right\| \cdot\left\|v \mid \mathcal{K}_{a-1, p}^{m-1}(D)\right\|
$$

holds for all $u \in \mathcal{K}_{a+1, p}^{m+1}(D)$ and $v \in \mathcal{K}_{a-1, p}^{m-1}(D)$.

Our main tool when investigating the Besov regularity of solutions to the PDEs will be the following embedding result between Kondratiev and Besov spaces, which is an extension of [30, Thm. 1]. A proof may be found in [40, Thm. 1.4.12].

Theorem 2 (Embeddings between Kondratiev and Besov spaces) Let $D \subset \mathbb{R}^{3}$ be some polyhedral type domain and assume $k \in \mathbb{N}_{0}, 0<q \leq \infty$. Furthermore, let $s, a \in \mathbb{R}$, $\gamma \in \mathbb{N}_{0}$, and suppose $\min (s, a)>\frac{\delta}{3} \gamma$, where $\delta$ denotes the dimension of the singular set (i.e., $\delta=0$ if there are only vertex singularities and $\delta=1$ if there are edge and vertex singularities). Then there exists some $0<\tau_{0} \leq p$ such that

$$
W_{q}^{k}\left([0, T], \mathcal{K}_{p, a}^{\gamma}(D)\right) \cap W_{q}^{k}\left([0, T], B_{p, \infty}^{s}(D)\right) \hookrightarrow W_{q}^{k}\left([0, T], B_{\tau, \infty}^{\gamma}(D)\right)
$$

for all $\tau_{*}<\tau<\tau_{0}$, where $\frac{1}{\tau_{*}}=\frac{\gamma}{3}+\frac{1}{p}$.

\section{Parabolic PDEs and Operator Pencils}

In the sequel we deal with two different parabolic settings, Problems 1 and 2, which are of general order and defined on domains of polyhedral type according to Definition 1. In particular, Problem 2 is the nonlinear version of Problem 1 and we investigate the spatial Besov regularity of the solutions of these two problems and to some extent also the Hölder regularity with respect to the time variable of Problem 1.

\subsection{The Fundamental Parabolic Problems}

Let $D$ denote some domain of polyhedral type in $\mathbb{R}^{d}$ according to Definition 1 with faces $\Gamma_{j}, j=1, \ldots, n$. For $0<T<\infty$ put $D_{T}=(0, T] \times D$ and $\Gamma_{j, T}=[0, T] \times \Gamma_{j}$.

We will investigate the Besov regularity of the following linear parabolic problem.

Problems 1 (Linear parabolic problem in divergence form) Let $m \in \mathbb{N}$. We consider the following first initial-boundary value problem

$$
\left\{\begin{aligned}
\frac{\partial}{\partial t} u+(-1)^{m} L\left(t, x, D_{x}\right) u & =f \text { in } D_{T}, \\
\left.\frac{\partial^{k-1} u}{\partial v^{k-1}}\right|_{\Gamma_{j, T}} & =0, \quad k=1, \ldots, m, j=1, \ldots, n, \\
\left.u\right|_{t=0} & =0 \text { in } D .
\end{aligned}\right\}
$$


Here $f$ is a function given on $D_{T}, v$ denotes the exterior normal to $\Gamma_{j, T}$, and the partial differential operator $L$ is given by

$$
L\left(t, x, D_{x}\right)=\sum_{|\alpha|,|\beta|=0}^{m} D_{x}^{\alpha}\left(a_{\alpha \beta}(t, x) D_{x}^{\beta}\right)
$$

where $a_{\alpha \beta}$ are bounded real-valued functions from $C^{\infty}\left(D_{T}\right)$ with $a_{\alpha \beta}=(-1)^{|\alpha|+|\beta|} a_{\beta \alpha}$. Furthermore, the operator $L$ is assumed to be uniformly elliptic with respect to $t \in[0, T]$, i.e.,

$$
\sum_{|\alpha|,|\beta|=m} a_{\alpha \beta} \xi^{\alpha} \xi^{\beta} \geq c|\xi|^{2 m} \quad \text { for all }(t, x) \in D_{T}, \quad \xi \in \mathbb{R}^{d}
$$

Let us denote by

$$
B(t, u, v)=\int_{D} \sum_{|\alpha|,|\beta|=0}^{m} a_{\alpha \beta}(t, x)\left(D_{x}^{\beta} u\right)\left(D_{x}^{\alpha} v\right) \mathrm{d} x
$$

the time-dependent bilinear form.

Moreover, for simplicity we set

$$
B_{\partial_{t^{k}}}(t, u, v)=\sum_{|\alpha|,|\beta| \leq m} \int_{D} \frac{\partial a_{\alpha \beta}(t, x)}{\partial t^{k}}\left(\mathrm{D}_{x}^{\beta} u\right)(t, x)\left(\mathrm{D}_{x}^{\alpha} v\right)(t, x) \mathrm{d} x .
$$

Remark 2 (Assumptions on the time-dependent bilinear form) When dealing with parabolic problems it will be reasonable to suppose that $B(t, \cdot, \cdot)$ satisfies

$$
B(t, u, u) \geq \mu\left\|u \mid H^{m}(D)\right\|^{2}
$$

for all $u \in \stackrel{\circ}{H}^{m}(D)$ and a.e. $t \in[0, T]$. We refer to [40, Rem. 2.3.5] for a detailed discussion.

It is our intention to also study nonlinear versions of Problem 1. Therefore, we modify (3.1) as follows.

Problems 2 (Nonlinear parabolic problem in divergence form) Let $m, M \in \mathbb{N}$ and $\varepsilon>0$. We consider the following nonlinear parabolic problem

$$
\left\{\begin{aligned}
\frac{\partial}{\partial t} u+(-1)^{m} L\left(t, x, D_{x}\right) u+\varepsilon u^{M} & =f \text { in } D_{T}, \\
\left.\frac{\partial^{k-1} u}{\partial \nu^{k-1}}\right|_{\Gamma_{j, T}} & =0, k=1, \ldots, m, j=1, \ldots, n, \\
\left.u\right|_{t=0} & =0 \text { in } D .
\end{aligned}\right\}
$$



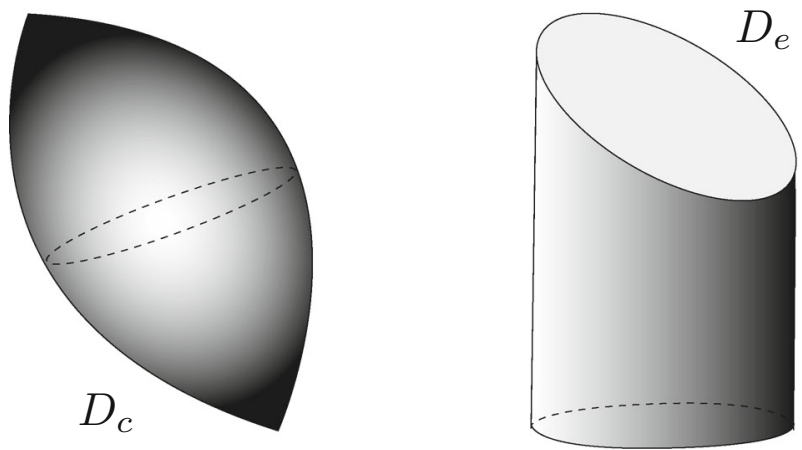

Fig. 2 Corner domain $D_{c}(l=0)$ and edge domain $D_{e}\left(l^{\prime}=0\right)$

The assumptions on $f$ and the operator $L$ are as in Problem 1. When we establish Besov regularity results for Problem 2 we interpret (3.6) as a fixed point problem and show that the regularity estimates for Problem 1 carry over to Problem 2, provided that $\varepsilon$ is sufficiently small.

\subsection{Operator Pencils}

In order to correctly state the global regularity results in Kondratiev spaces for Problems 1 and 2, we need to work with operator pencils generated by the corresponding elliptic problems in the polyhedral type domain $D \subset \mathbb{R}^{3}$.

We briefly recall the basic facts needed in the sequel. For further information on this subject we refer to [33] and [36, Sects. 2.3, 3.2, 4.1]. On a domain $D \subset \mathbb{R}^{3}$ of polyhedral type according to Definition 1 we consider the problem

$$
\left\{\begin{aligned}
L u & =f \text { in } D, \\
\left.\frac{\partial^{k-1} u}{\partial v^{k-1}}\right|_{\partial D} & =0, \quad k=1, \ldots, m .
\end{aligned}\right\}
$$

The singular set $S$ of $D$ then is given by the boundary points $M_{1} \cup \ldots \cup M_{l} \cup$ $\left\{x^{(1)}, \ldots, x^{\left(l^{\prime}\right)}\right\}$. We do not exclude the cases $l=0$ (corner domain) and $l^{\prime}=0$ (edge domain). In the last case, the set $S$ consists only of smooth nonintersecting edges. Figure 2 gives examples of polyhedral domains without edges or corners, respectively.

The elliptic boundary value problem (3.7) on $D$ generates two types of operator pencils for the edges $M_{k}$ and for the vertices $x^{(i)}$ of the domain, respectively.

(1) Operator pencil $A_{\xi}(\lambda)$ for edge points

The pencils $A_{\xi}(\lambda)$ for edge points $\xi \in M_{k}$ are defined as follows: According to Definition 1 there exists a neighborhood $U_{\xi}$ of $\xi$ and a diffeomorphism $\kappa_{\xi}$ mapping $D \cap U_{\xi}$ onto $\mathcal{D}_{\xi} \cap B_{1}(0)$, where $\mathcal{D}_{\xi}$ is a dihedron (Fig. 3).

Let $\Gamma_{k_{ \pm}}$be the faces adjacent to $M_{k}$. Then by $\mathcal{D}_{\xi}$ we denote the dihedron which is bounded by the half-planes $\stackrel{\circ}{\Gamma}_{k_{ \pm}}$tangent to $\Gamma_{k_{ \pm}}$at $\xi$ and the edge $M_{\xi}=\stackrel{\circ}{\Gamma}_{k_{+}} \cap \stackrel{\circ}{\Gamma}_{k_{-}}$. 
Fig. 3 Dihedron $\mathcal{D}_{\xi}$

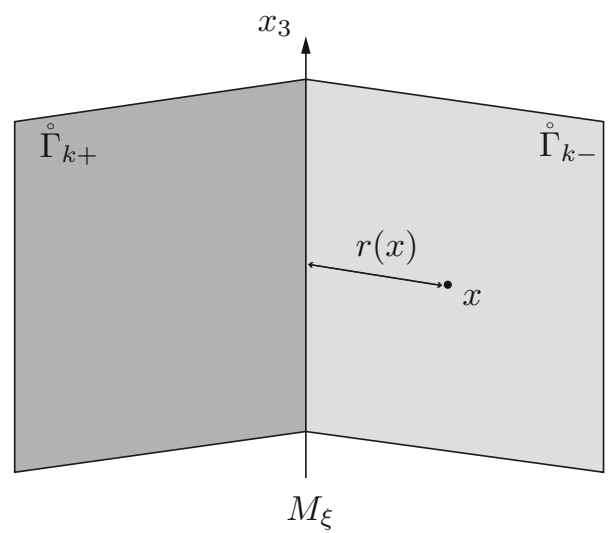

Furthermore, let $r, \varphi$ be polar coordinates in the plane perpendicular to $M_{\xi}$ such that

$$
\stackrel{\circ}{\Gamma}_{k_{ \pm}}=\left\{x \in \mathbb{R}^{3}: r>0, \varphi= \pm \frac{\theta_{\xi}}{2}\right\}
$$

We define the operator pencil $A_{\xi}(\lambda)$ as follows:

$$
A_{\xi}(\lambda) U(\varphi)=r^{2 m-\lambda} L_{0}\left(0, D_{x}\right) u
$$

where $u(x)=r^{\lambda} U(\varphi), \lambda \in \mathbb{C}, U$ is a function on $I_{\xi}:=\left(\frac{-\theta_{\xi}}{2}, \frac{\theta_{\xi}}{2}\right)$, and

$$
L_{0}\left(\xi, D_{x}\right)=\sum_{|\alpha|=|\beta|=m} D_{x}^{\alpha}\left(a_{\alpha \beta}(\xi) D_{x}^{\beta}\right)
$$

denotes the main part of the differential operator $L\left(x, D_{x}\right)$ with coefficients frozen at $\xi$. This way we obtain in (3.8) a boundary value problem for the function $U$ on the 1-dimensional subdomain $I_{\xi}$ with the complex parameter $\lambda$. Obviously, $A_{\xi}(\lambda)$ is a polynomial of degree $2 m$ in $\lambda$.

The operator $A_{\xi}(\lambda)$ realizes a continuous mapping

$$
H^{2 m}\left(I_{\xi}\right) \rightarrow L_{2}\left(I_{\xi}\right)
$$

for every $\lambda \in \mathbb{C}$. Furthermore, $A_{\xi}(\lambda)$ is an isomorphism for all $\lambda \in \mathbb{C}$ with the possible exception of a denumerable set of isolated points, the spectrum of $A_{\xi}(\lambda)$, which consists of its eigenvalues with finite algebraic multiplicities: Here a complex number $\lambda_{0}$ is called an eigenvalue of the pencil $A_{\xi}(\lambda)$ if there exists a nonzero function $U \in H^{2 m}\left(I_{\xi}\right)$ such that $A_{\xi}\left(\lambda_{0}\right) U=0$. It is known that the 'energy line' $\operatorname{Re} \lambda=m-1$ does not contain eigenvalues of the pencil $A_{\xi}(\lambda)$. We denote by $\delta_{ \pm}^{(\xi)}$ the largest positive real numbers such that the strip

$$
m-1-\delta_{-}^{(\xi)}<\operatorname{Re} \lambda<m-1+\delta_{+}^{(\xi)}
$$


is free of eigenvalues of the pencil $A_{\xi}(\lambda)$. Furthermore, we put

$$
\delta_{ \pm}^{(k)}=\inf _{\xi \in M_{k}} \delta_{ \pm}^{(\xi)}, \quad k=1, \ldots, l
$$

For example, concerning the Dirichlet problem for the Poisson equation on a domain $D \subset \mathbb{R}^{3}$ of polyhedral type, the eigenvalues of the pencil $A_{\xi}(\lambda)$ are given by

$$
\lambda_{k}=k \pi / \theta_{\xi}, \quad k= \pm 1, \pm 2, \ldots,
$$

where $\theta_{\xi}$ is the inner angle at the edge point $\xi$, cf. [40, Ex. 2.5.2]. Therefore, the first positive eigenvalue is $\lambda_{1}=\frac{\pi}{\theta_{\xi}}$ and we obtain $\delta_{ \pm}=\frac{\pi}{\theta_{\xi}}$, cf. [40, Ex. 2.5.1].

(2) Operator pencil $\mathfrak{A}_{i}(\lambda)$ for corner points

Let $x^{(i)}$ be a vertex of $D$. According to Definition 1 there exists a neighborhood $U_{i}$ of $x^{(i)}$ and a diffeomorphism $\kappa_{i}$ mapping $D \cap U_{i}$ onto $K_{i} \cap B_{1}(0)$, where

$$
K_{i}=\left\{x \in \mathbb{R}^{3}: x /|x| \in \Omega_{i}\right\}
$$

is a polyhedral cone with edges and vertex at the origin. W.l.o.g. we may assume that the Jacobian matrix $\kappa_{i}^{\prime}(x)$ is equal to the identity matrix at the point $x^{(i)}$. We introduce spherical coordinates $\rho=|x|, \omega=\frac{x}{|x|}$ in $K_{i}$ and define the operator pencil

$$
\mathfrak{A}_{i}(\lambda) U(\omega)=\rho^{2 m-\lambda} L_{0}\left(x^{(i)}, D_{x}\right) u
$$

where $u(x)=\rho^{\lambda} U(\omega)$ and $U \in \stackrel{\circ}{H}^{m}\left(\Omega_{i}\right)$ is a function on $\Omega_{i}$. An eigenvalue of $\mathfrak{A}_{i}(\lambda)$ is a complex number $\lambda_{0}$ such that $\mathfrak{A}_{i}\left(\lambda_{0}\right) U=0$ for some nonzero function $U \in \stackrel{\circ}{H}^{m}\left(\Omega_{i}\right)$. The operator $\mathfrak{A}_{i}(\lambda)$ realizes a continuous mapping

$$
\stackrel{\circ}{H}^{m}\left(\Omega_{i}\right) \rightarrow H^{-m}\left(\Omega_{i}\right)
$$

Furthermore, it is known that $\mathfrak{A}_{i}(\lambda)$ is an isomorphism for all $\lambda \in \mathbb{C}$ with the possible exception of a denumerable set of isolated points. The mentioned enumerable set consists of eigenvalues with finite algebraic multiplicities.

Moreover, the eigenvalues of $\mathfrak{A}_{i}(\lambda)$ are situated, except for finitely many, outside a double sector $|\operatorname{Re} \lambda|<\varepsilon|\operatorname{Im} \lambda|$ containing the imaginary axis, cf. [33, Thm. 10.1.1]. In Fig. 4 the situation is illustrated: Outside the yellow area there are only finitely many eigenvalues of the operator pencil $\mathfrak{A}_{i}(\lambda)$.

Dealing with regularity properties of solutions, we look for the widest strip in the $\lambda$-plane, free of eigenvalues and containing the 'energy line' $\operatorname{Re} \lambda=m-3 / 2$, cf. Assumption 1. From what was outlined above, information on the width of this strip is obtained from lower estimates for real parts of the eigenvalues situated over the energy line.

Remark 3 (Operator pencils for parabolic problems) Since we study parabolic PDEs, where the differential operator $L\left(t, x, D_{x}\right)$ additionally depends on the time $t$, we have 
Fig. 4 Eigenvalues of operator pencil $\mathfrak{A}_{i}(\lambda)$

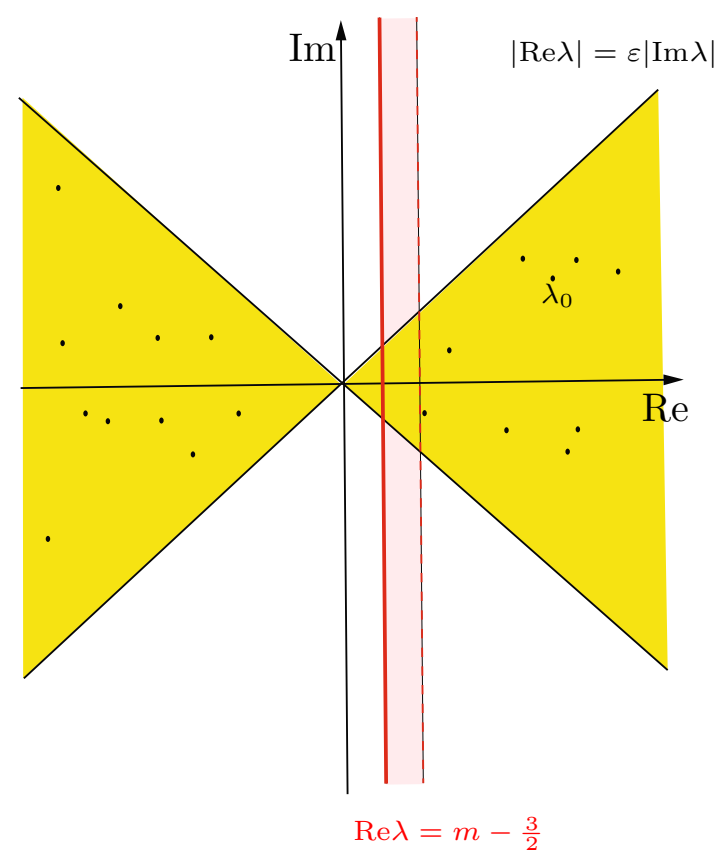

to work with operator pencils $A_{\xi}(\lambda, t)$ and $\mathfrak{A}_{i}(\lambda, t)$ in this context. The philosophy is to fix $t \in[0, T]$ and define the pencils as above: We replace (3.8) by

$$
A_{\xi}(\lambda, t) U(\varphi)=r^{2 m-\lambda} L_{0}\left(t, 0, D_{x}\right) u,
$$

and work with $\delta_{ \pm}^{(\xi)}(t)$ and $\delta_{ \pm}^{(k)}(t)=\inf _{\xi \in M_{k}} \delta_{ \pm}^{(\xi)}(t)$ in (3.9) and (3.10), respectively. Moreover, we put

$$
\delta_{ \pm}^{(k)}=\inf _{t \in[0, T]} \delta_{ \pm}^{(k)}(t), \quad k=1, \ldots, l
$$

Similar for $\mathfrak{A}_{i}(\lambda, t)$, where now (3.11) is replaced by

$$
\mathfrak{A}_{i}(\lambda, t) U(\omega)=\rho^{2 m-\lambda} L_{0}\left(t, x^{(i)}, D_{x}\right) u .
$$

\section{Regularity Results in Sobolev and Kondratiev Spaces}

This section presents regularity results for Problems 1 and 2 in Sobolev and Kondratiev spaces. They will form the basis for obtaining regularity results in Besov spaces later on via suitable embeddings. The results in Sobolev and Kondratiev spaces for Problems 1 and 2 on domains of polyhedral type $D \subset \mathbb{R}^{d}$ are essentially new and not published elsewhere so far: In [22] we restricted our investigations to polyhedral cones $K \subset \mathbb{R}^{3}$ relying on the results from [35]. 
However, the extension of the regularity results for Problem 1 to polyhedral type domains follows from very similar arguments as in [22], which is why we merely state the results in Sects. 4.1 and 4.2 and give references for the proofs wherever necessary. In contrast to this the regularity results for the nonlinear Problem 2 require some careful adaptations and are carried out in detail in Sect. 4.3.

\subsection{Regularity Results in Sobolev Spaces for Problem I}

In this subsection, we are concerned with the Sobolev regularity of the weak solution of Problem 1. We start with the following lemma, whose proof is similar to [5, Lem. 4.1].

Lemma 1 (Continuity of bilinear form) Assume that for each $t \in[0, T], F(t, \cdot, \cdot)$ : $\stackrel{\circ}{H}^{m}(D) \times \stackrel{\circ}{H}^{m}(D) \rightarrow \mathbb{R}$ is a bilinear map satisfying

$$
|F(t, u, v)| \leq C\left\|u\left|\stackrel{\circ}{H}^{m}(D)\|\| v\right| \stackrel{\circ}{H}^{m}(D)\right\|
$$

for all $t \in[0, T]$ and all $u, v \in \stackrel{\circ}{H}^{m}(D)$, where $C$ is a constant independent of $u, v$, and t. Assume further that $F(\cdot, u, v)$ is measurable on $[0, T]$ for each pair $u, v \in \stackrel{\circ}{H}^{m}(D)$. Assume that $u \in H^{m, 1 *}\left(D_{T}\right)$ satisfies $u(0) \equiv 0$ and

$$
\left(\partial_{t} u(t), v\right)+B(t, u(t), v)=\int_{0}^{t} F(\tau, u(\tau), v) \mathrm{d} \tau
$$

for a.e. $t \in[0, T]$ and all $v \in \stackrel{\circ}{H}^{m}(D)$. Then $u \equiv 0$ on $[0, T] \times D$.

Using the spectral method the following regularity result now follows.

Theorem 3 (Sobolev regularity without time derivatives) Let $f \in L_{2}\left([0, T], H^{-m}(D)\right)$. Then Problem 1 has a unique weak solution $u$ in the space $H^{m, 1 *}\left(D_{T}\right)$ and the following estimate holds

$$
\left\|u\left|H^{m, 1 *}\left(D_{T}\right)\|\leq C\| f\right| L_{2}\left([0, T], H^{-m}(D)\right)\right\|,
$$

where $C$ is a constant independent of $f$ and $u$.

This proof follows [5, Lem. 4.2], which in turn is based on [26, Sect. 7.1.2].

By an application of Theorem 3 and induction we obtain the following regularity result. The proof is similar to [6, Thm. 2].

Theorem 4 (Sobolev regularity with time derivatives) Let $l \in \mathbb{N}_{0}$ and assume that the right-hand side $f$ of Problem 1 satisfies

$$
f \in H^{l}\left([0, T], H^{-m}(D)\right) \quad \text { and } \quad \partial_{t^{k}} f(x, 0)=0 \quad \text { for } \quad k=0, \ldots, l-1 .
$$

Then the weak solution $u$ in the space $H^{m, 1 *}\left(D_{T}\right)$ of Problem 1 in fact belongs to $H^{m, l+1 *}\left(D_{T}\right)$, i.e., has derivatives with respect to $t$ up to order l satisfying

$$
\partial_{t^{k}} u \in H^{m, 1 *}\left(D_{T}\right) \text { for } k=0, \ldots, l,
$$


and

$$
\sum_{k=0}^{l}\left\|\partial_{t^{k}} u\left|H^{m, 1 *}\left(D_{T}\right)\left\|\leq C \sum_{k=0}^{l}\right\| \partial_{t^{k}} f\right| L_{2}\left([0, T], H^{-m}(D)\right)\right\|,
$$

where $C$ is a constant independent of $u$ and $f$.

Remark 4 Note that the regularity results for the solution $u$ in [35, Thm. 2.1., Lem. 3.1] are slightly stronger than the ones obtained in Theorem 4 above (with the cost of also assuming more regularity on the right-hand side $f$ ). By using similar arguments as in [5, Lem. 4.3] we are probably able to also show in our context that Theorem 3 can be strengthened in the sense that if $f \in L_{2}\left([0, T], L_{2}(D)\right)$ then the weak solution $u$ of Problem 1 belongs in fact to $L_{2}\left([0, T], \stackrel{\circ}{H}^{m}\right) \cap H^{1}\left([0, T], L_{2}(D)\right)$. A corresponding generalization of Theorem 4 should also be possible in the spirit of [5, Thm. 3.1]. However, for our purposes the above results on the Sobolev regularity are sufficient, so these investigations are postponed for the time being.

\subsection{Regularity Results in Kondratiev Spaces for Problem I}

Concerning weighted Sobolev regularity of Problem 1 first fundamental results on polyhedral cones $K \subset \mathbb{R}^{3}$ can be found in [35, Thms. 3.3, 3.4]. In [22] we extended and generalized these results, which we now wish to transfer to our setting of polyhedral type domains $D \subset \mathbb{R}^{3}$.

For our regularity assertions we rely on known results for elliptic equations. Therefore, we consider first the following Dirichlet problem for elliptic equations

$$
\left\{\begin{array}{ll}
L u=F & \text { on } \quad D, \\
\left.\frac{\partial^{k} u}{\partial \nu^{k}}\right|_{\Gamma_{j}}=0, & k=1, \ldots, m, j=1, \ldots, n,
\end{array}\right\}
$$

where $D \subset \mathbb{R}^{3}$ is a domain of polyhedral type according to Definition 1 with faces $\Gamma_{j}$. Moreover, we assume that

$$
L\left(x, D_{x}\right)=\sum_{|\alpha| \leq 2 m} A_{\alpha}(x) D_{x}^{\alpha}
$$

is a uniformly elliptic differential operator of order $2 m$ with smooth coefficients $A_{\alpha}$. We need the following technical assumptions in order to state the Kondratiev regularity of (4.4).

Assumption 1 (Assumptions on operator pencils) Consider the operator pencils $\mathfrak{A}_{i}(\lambda, t), i=1, \ldots, l^{\prime}$ for the vertices and $A_{\xi}(\lambda, t)$ with $\xi \in M_{k}, k=1, \ldots, l$ for the edges of the polyhedral type domain $D \subset \mathbb{R}^{3}$ introduced in Sect. 3.2. For the elliptic problem (4.4) we may drop $t$ from the notation of the pencils, otherwise (for our parabolic problems) we assume $t \in[0, T]$ is fixed. 
Let $\mathcal{K}_{p, b}^{\gamma}(D)$ and $\mathcal{K}_{p, b^{\prime}}^{\gamma^{\prime}}(D)$ be two Kondratiev spaces, where the singularity set $S$ of $D$ is given by $S=M_{1} \cup \cdots \cup M_{l} \cup\left\{x^{(1)}, \ldots, x^{\left(l^{\prime}\right)}\right\}$ and weight parameters $b, b^{\prime} \in \mathbb{R}$. Then we assume that the closed strip between the lines

$$
\operatorname{Re} \lambda=b+2 m-\frac{3}{2} \quad \text { and } \quad \operatorname{Re} \lambda=b^{\prime}+2 m-\frac{3}{2}
$$

does not contain eigenvalues of $\mathfrak{A}_{i}(\lambda, t)$. Moreover, $b$ and $b^{\prime}$ satisfy

$$
-\delta_{-}^{(k)}<b+m<\delta_{+}^{(k)}, \quad-\delta_{-}^{(k)}<b^{\prime}+m<\delta_{+}^{(k)}, \quad k=1, \ldots, l,
$$

where $\delta_{ \pm}^{(k)}$ are defined in (3.10) (replaced by (3.12) for parabolic problems).

Remark 5 If $l^{\prime}=0$ we have an edge domain without vertices, cf. Fig. 2. In this case condition (4.5) is empty. Moreover, if $l=0$, we have a corner domain without edges, in which case condition (4.6) is empty. For further remarks and explanations concerning Assumption 1 we refer to [22, Rem. 3.3].

The following lemma on the regularity of solutions to elliptic boundary value problems in domains of polyhedral type is taken from [36, Cor. 4.1.10, Thm. 4.1.11]. We rewrite it for our scale of Kondratiev spaces.

Lemma 2 (Kondratiev regularity for elliptic PDEs) Let $D \subset \mathbb{R}^{3}$ be a domain of polyhedral type. Moreover, let $u \in \mathcal{K}_{2, a+2 m}^{\gamma}(D)$ be a solution of (4.4), where

$$
F \in \mathcal{K}_{2, a}^{\gamma-2 m}(D) \cap \mathcal{K}_{2, a^{\prime}}^{\gamma^{\prime}-2 m}(D), \quad \gamma \geq m, \quad \gamma^{\prime} \geq m
$$

Suppose that $\mathcal{K}_{2, a}^{\gamma}(D)$ and $\mathcal{K}_{2, a^{\prime}}^{\gamma^{\prime}}(D)$ satisfy Assumption 1. Then $u \in \mathcal{K}_{2, a^{\prime}+2 m}^{\gamma^{\prime}}(D)$ and

$$
\left\|u\left|\mathcal{K}_{2, a^{\prime}+2 m}^{\gamma^{\prime}}(D)\|\leq C\| F\right| \mathcal{K}_{2, a^{\prime}}^{\gamma^{\prime}-2 m}(D)\right\|,
$$

where $C$ is a constant independent of $u$ and $F$.

Remark 6 In particular, if in Theorem 4 we use the stronger assumption $\partial_{t^{k}} f(t) \in$ $L_{2}(D)$ instead of $\partial_{t^{k}} f(t) \in H^{-m}(D)$ for $k=0, \ldots, l$, then it follows that

$$
\partial_{t^{k}} f(t) \in L_{2}(D)=\mathcal{K}_{2,0}^{0}(D) \hookrightarrow \mathcal{K}_{2,-m}^{-m}(D),
$$

where the latter embedding follows from the corresponding duality assertion, i.e., we have $\mathcal{K}_{2, m}^{m}(D) \hookrightarrow \mathcal{K}_{2,0}^{0}(D)$ since $m \geq 0$. In this case, the solution $u$ of Problem 1 satisfies

$$
\partial_{t^{k}} u(t) \in \stackrel{\circ}{H}^{m}(D) \hookrightarrow \stackrel{\circ}{\mathcal{K}}_{2, m}^{m}(D) \hookrightarrow \mathcal{K}_{2, a}^{0}(D), \quad a \leq m,
$$


where the first embedding is taken from [36, Lem. 3.1.6] and the second embedding for Kondratiev spaces holds whenever $m \geq a$. We additionally require in our considerations that $\partial_{t^{k}} u(t) \in \mathcal{K}_{2, a}^{0}(D) \hookrightarrow \mathcal{K}_{2,-m}^{-m}(D)$ which holds for $a \geq-m$. From (4.7) and (4.8) we see that it is possible to take $\gamma=m$ and $a=-m$ in Lemma 2, i.e., if $f(t) \in \mathcal{K}_{2,-m}^{-m}(D)$ then $u(t) \in \mathcal{K}_{2, m}^{m}(D)$. Note that all our arguments with $u(t)$ and $f(t)$, respectively, hold for a.e. $t \in[0, T]$. However, since lower order time derivatives are continuous w.r.t. suitable spaces (but not necessarily the highest one, cf. the proof of Thm. 11), we will suppress this distinction in the sequel.

Using similar arguments as in [35, Thm. 3.3] we are now able to show the following regularity result in Kondratiev spaces. The proof follows along the same lines as [22, Thm. 3.6].

Theorem 5 (Kondratiev regularity A) Let $D \subset \mathbb{R}^{3}$ be a domain of polyhedral type. Let $\gamma \in \mathbb{N}$ with $\gamma \geq 2 m$ and put $\gamma_{m}:=\left[\frac{\gamma-1}{2 m}\right]$. Furthermore, let $a \in \mathbb{R}$ with $a \in[-m, m]$. Assume that the right-hand side $f$ of Problem 1 satisfies

(i) $\partial_{t^{k}} f \in L_{2}\left(D_{T}\right) \cap L_{2}\left([0, T], \mathcal{K}_{2, a+2 m\left(\gamma_{m}-k\right)}^{2 m\left(\gamma_{m}-k\right)}(D)\right), \quad k=0, \ldots, \gamma_{m} ; \quad \partial_{t \gamma_{m}+1} f \in$ $L_{2}\left(D_{T}\right)$.

(ii) $\partial_{t^{k}} f(x, 0)=0, \quad k=0,1, \ldots, \gamma_{m}$.

Furthermore, let Assumption 1 hold for weight parameters $b=a+2 m\left(\gamma_{m}-i\right)$, where $i=0, \ldots, \gamma_{m}$, and $b^{\prime}=-m$. Then for the weak solution $u \in H^{m, \gamma_{m}+2 *}\left(D_{T}\right)$ of Problem 1 we have

$$
\partial_{t^{l+1}} u \in L_{2}\left([0, T], \mathcal{K}_{2, a+2 m\left(\gamma_{m}-l\right)}^{2 m\left(\gamma_{m}-l\right)}(D)\right)
$$

for $l=-1,0, \ldots, \gamma_{m}$. In particular, for the derivatives $\partial_{t^{l+1}} u$ up to order $\gamma_{m}+1$ we have the a priori estimate

$$
\begin{aligned}
& \sum_{l=-1}^{\gamma_{m}}\left\|\partial_{t^{l+1}} u \mid L_{2}\left([0, T], \mathcal{K}_{2, a+2 m\left(\gamma_{m}-l\right)}^{2 m\left(\gamma_{m}-l\right)}(D)\right)\right\| \\
& \quad \lesssim \sum_{k=0}^{\gamma_{m}}\left\|\partial_{t^{k}} f\left|L_{2}\left([0, T], \mathcal{K}_{2, a+2 m\left(\gamma_{m}-k\right)}^{2 m\left(\gamma_{m}-k\right)}(D)\right)\left\|+\sum_{k=0}^{\gamma_{m}+1}\right\| \partial_{t^{k}} f\right| L_{2}\left(D_{T}\right)\right\|,
\end{aligned}
$$

where the constant is independent of $u$ and $f$.

Remark 7 The existence of the solution $u \in H^{m, \gamma_{m}+2 *}\left(D_{T}\right)$ follows from Theorem 4 using $l=\gamma_{m}+1$.

The regularity results obtained in Theorem 5 only hold under certain restrictions on the parameter $a$ we are able to choose. In particular, we cannot choose $\gamma_{m}>0$ if we have a nonconvex polyhedral type domains $D$, since there is no suitable $a$ satisfying all of our requirements in this case. In order to treat nonconvex domains as well, we impose stronger assumptions on the right-hand side $f$, requiring that it is arbitrarily smooth w.r.t. the time. This additional assumption allows for a larger range of $a$. 
However, as a drawback, these results are hard to apply to nonlinear equations since the right-hand sides are not taken from a Banach or quasi-Banach space. The proof of the following theorem is similar to [22, Thm. 3.9] adapted to our setting.

Theorem 6 (Kondratiev regularity B) Let $D \subset \mathbb{R}^{3}$ be a domain of polyhedral type and $\eta \in \mathbb{N}$ with $\eta \geq 2 m$. Moreover, let $l \in \mathbb{N}_{0}$ and $a \in \mathbb{R}$ with $a \in[-m, m]$. Assume that the right-hand side $f$ of Problem 1 satisfies

(i) $f \in \bigcap_{l=0}^{\infty} H^{l}\left([0, T], L_{2}(D) \cap \mathcal{K}_{2, a}^{\eta-2 m}(D)\right)$.

(ii) $\partial_{t^{l}} f(x, 0)=0, \quad l \in \mathbb{N}_{0}$.

Furthermore, let Assumption 1 hold for weight parameters $b=a$ and $b^{\prime}=-m$. Then for the weak solution $u \in \bigcap_{l=0}^{\infty} H^{m, l+1 *}\left(D_{T}\right)$ of Problem 1 we have

$$
\partial_{t^{l}} u \in L_{2}\left([0, T], \mathcal{K}_{2, a+2 m}^{\eta}(D)\right) \quad \text { for all } l \in \mathbb{N}_{0}
$$

In particular, for the derivative $\partial_{t} l u$ we have the a priori estimate

$$
\begin{aligned}
& \sum_{k=0}^{l}\left\|\partial_{t^{k}} u \mid L_{2}\left([0, T], \mathcal{K}_{2, a+2 m}^{\eta}(D)\right)\right\| \\
& \quad \lesssim \sum_{k=0}^{l+(\eta-2 m)}\left\|\partial_{t^{k}} f\left|L_{2}\left([0, T], \mathcal{K}_{2, a}^{\eta-2 m}(D)\right)\left\|+\sum_{k=0}^{l+1+(\eta-2 m)}\right\| \partial_{t^{k}} f\right| L_{2}\left(D_{T}\right)\right\|,
\end{aligned}
$$

where the constant is independent of $u$ and $f$.

Remark 8 In Theorem 6 compared to Theorem 5 we only require the parameter $a$ to satisfy $a \in[-m, m]$ and $-\delta_{-}^{(k)}<a+m<\delta_{+}^{(k)}$ independent of the regularity parameter $\eta$ which can be arbitrarily high. In particular, for the heat equation on a domain of polyhedral type $D$ (which for simplicity we assume to be a polyhedron with straight edges and faces where $\theta_{k}$ denotes the angle at the edge $M_{k}$ ), we have $\delta_{ \pm}^{(k)}=\frac{\pi}{\theta_{k}}$, which leads to the restriction $-1 \leq a<\min \left(1, \frac{\pi}{\theta_{k}}-1\right)$. Therefore, even in the extremal case when $\theta_{k}=2 \pi$ we can still take $-1 \leq a<-\frac{1}{2}$ (resulting in $u \in L_{2}\left([0, T], \mathcal{K}_{a+2}^{\eta}(D)\right)$ being locally integrable since $\left.a+2>0\right)$. Then choosing $\eta$ arbitrary high, we also cover nonconvex polyhedral type domains with our results from Theorem 6.

\subsection{Regularity Results in Sobolev and Kondratiev Spaces for Problem II}

In this subsection, we show that the regularity estimates in Kondratiev and Sobolev spaces as stated in Theorems 5 and 4, respectively, carry over to Problem 2, provided that $\varepsilon$ is sufficiently small. In order to do this we interpret Problem 2 as a fixed point problem in the following way. 
Let $\widetilde{\mathcal{D}}$ and $S$ be Banach spaces ( $\widetilde{\mathcal{D}}$ and $S$ will be specified in the theorem below) and let $\tilde{L}^{-1}: \widetilde{\mathcal{D}} \rightarrow S$ be the linear operator defined via

$$
\tilde{L} u:=\frac{\partial}{\partial t} u+(-1)^{m} L u .
$$

Problem 2 is equivalent to

$$
\tilde{L} u=f-\varepsilon u^{M}=: N u,
$$

where $N: S \rightarrow \widetilde{\mathcal{D}}$ is a nonlinear operator. If we can show that $N$ maps $S$ into $\widetilde{\mathcal{D}}$, then a solution of Problem 2 is a fixed point of the problem

$$
\left(\tilde{L}^{-1} \circ N\right) u=u \text {. }
$$

Our aim is to apply Banach's fixed point theorem, which will also guarantee uniqueness of the solution, if we can show that $T:=\left(\tilde{L}^{-1} \circ N\right): S_{0} \rightarrow S_{0}$ is a contraction mapping, i.e., there exists some $q \in[0,1)$ such that

$$
\|T(x)-T(y)|S\|\leq q\| x-y| S\| \text { for all } x, y \in S_{0},
$$

where the corresponding subset $S_{0} \subset S$ is a small closed ball with center $\tilde{L}^{-1} f$ (the solution of the corresponding linear problem) and suitably chosen radius $R>0$.

Our main result is stated in the theorem below.

Theorem 7 (Nonlinear Sobolev and Kondratiev regularity) Let $\tilde{L}$ and $N$ be as described above. Assume the assumptions of Theorem 5 are satisfied and, additionally, we have $\gamma_{m} \geq 1, m \geq 2$, and $a \geq-\frac{1}{2}$. Let

$$
\mathcal{D}_{1}:=\bigcap_{k=0}^{\gamma_{m}} H^{k}\left([0, T], \mathcal{K}_{2, a+2 m\left(\gamma_{m}-k\right)}^{2 m\left(\gamma_{m}-k\right)}(D)\right), \quad \mathcal{D}_{2}:=H^{\gamma_{m}+1}\left([0, T], L_{2}(D)\right)
$$

and consider the data space

$$
\widetilde{\mathcal{D}}:=\left\{f \in \mathcal{D}_{1} \cap \mathcal{D}_{2}: \partial_{t^{k}} f(0, \cdot)=0, \quad k=0, \ldots, \gamma_{m}\right\}
$$

Moreover, let

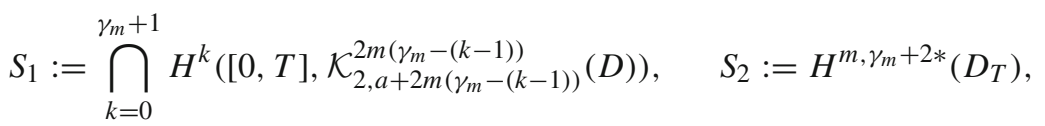

and consider the solution space $S:=S_{1} \cap S_{2}$. Suppose that $f \in \widetilde{\mathcal{D}}$ and put $\eta:=\|f \mid \widetilde{\mathcal{D}}\|$ and $r_{0}>1$. Moreover, we choose $\varepsilon>0$ so small that

$$
\eta^{2(M-1)}\left\|\tilde{L}^{-1}\right\|^{2 M-1} \leq \frac{1}{c \varepsilon M}\left(r_{0}-1\right)\left(\frac{1}{r_{0}}\right)^{2 M-1}, \quad \text { if } r_{0}\left\|\tilde{L}^{-1}\right\| \eta>1,
$$


and

$$
\left\|\tilde{L}^{-1}\right\|<\frac{r_{0}-1}{r_{0}}\left(\frac{1}{c \varepsilon M}\right), \quad \text { if } r_{0}\left\|\tilde{L}^{-1}\right\| \eta<1
$$

where $c>0$ denotes the constant in (4.28) resulting from our estimates below. Then there exists a unique solution $u \in S_{0} \subset S$ of Problem 2, where $S_{0}$ denotes a small ball around $\tilde{L}^{-1} f$ (the solution of the corresponding linear problem) with radius $R=\left(r_{0}-1\right) \eta\left\|\tilde{L}^{-1}\right\|$.

Proof Let $u$ be the solution of the linear problem $\tilde{L} u=f$. From Theorems 5 and 4 we know that $\tilde{L}^{-1}: \widetilde{\mathcal{D}} \rightarrow S$ is a bounded operator. If $u^{M} \in \widetilde{\mathcal{D}}$ (this will immediately follow from our calculations in Step 1 as explained in Step 2 below), the nonlinear part $N$ satisfies the desired mapping properties, i.e., $N u=f-\varepsilon u^{M} \in \widetilde{\mathcal{D}}$ and we can apply Theorem 5 now with right-hand side $N u$.

Step 1: Since

$$
\left(\tilde{L}^{-1} \circ N\right)(v)-\left(\tilde{L}^{-1} \circ N\right)(u)=\tilde{L}^{-1}\left(f-\varepsilon v^{M}\right)-\tilde{L}^{-1}\left(f-\varepsilon u^{M}\right)=\varepsilon \tilde{L}^{-1}\left(u^{M}-v^{M}\right)
$$

one sees that $\tilde{L}^{-1} \circ N$ is a contraction if, and only, if

$$
\varepsilon\left\|\tilde{L}^{-1}\left(u^{M}-v^{M}\right)|S\|\leq q\| u-v| S\right\| \quad \text { for some } \quad q<1,
$$

where $u, v \in S_{0}$ (meaning $u, v \in B_{R}\left(\tilde{L}^{-1} f\right)$ in $\left.S\right)$. We analyze the resulting condition with the help of the formula $u^{M}-v^{M}=(u-v) \sum_{j=0}^{M-1} u^{j} v^{M-1-j}$. This together with Theorem 5 gives

$$
\begin{aligned}
& \left\|\tilde{L}^{-1}\left(u^{M}-v^{M}\right) \mid S\right\| \\
& \leq\left\|\tilde{L}^{-1}\right\| \| u^{M}-v^{M}|\widetilde{\mathcal{D}}| \\
& =\left\|\tilde{L}^{-1}\right\|\left\|u^{M}-v^{M} \mid \mathcal{D}_{1} \cap \mathcal{D}_{2}\right\| \\
& =\left\|\tilde{L}^{-1}\right\|\left(\left\|u^{M}-v^{M}\left|\mathcal{D}_{1}\|+\| u^{M}-v^{M}\right| \mathcal{D}_{2}\right\|\right) \\
& =\left\|\tilde{L}^{-1}\right\|\left(\left\|(u-v) \sum_{j=0}^{M-1} u^{j} v^{M-1-j}\left|\mathcal{D}_{1}\|+\|(u-v) \sum_{j=0}^{M-1} u^{j} v^{M-1-j}\right| \mathcal{D}_{2}\right\|\right) \\
& \lesssim\left\|\tilde{L}^{-1}\right\|\left(\sum_{k=0}^{\gamma_{m}}\left\|\partial_{t^{k}}\left[(u-v) \sum_{j=0}^{M-1} u^{j} v^{M-1-j}\right] \mid L_{2}\left([0, T], \mathcal{K}_{2, a+2 m\left(\gamma_{m}-k\right)}^{2 m\left(\gamma_{m}-k\right)}(D)\right)\right\|\right. \\
& \left.+\sum_{k=0}^{\gamma_{m}+1}\left\|\partial_{t^{k}}\left[(u-v) \sum_{j=0}^{M-1} u^{j} v^{M-1-j}\right] \mid L_{2}\left(D_{T}\right)\right\|\right) \text {. }
\end{aligned}
$$


Concerning the derivatives, we use Leibniz's formula twice and we see that

$$
\begin{aligned}
\partial_{t^{k}}\left(u^{M}-v^{M}\right) & =\partial_{t^{k}}\left[(u-v) \sum_{j=0}^{M-1} u^{j} v^{M-1-j}\right] \\
& =\sum_{l=0}^{k}\left(\begin{array}{l}
k \\
l
\end{array}\right) \partial_{t^{l}}(u-v) \cdot \partial_{t^{k-l}}\left(\sum_{j=0}^{M-1} u^{j} v^{M-1-j}\right) \\
& =\sum_{l=0}^{k}\left(\begin{array}{l}
k \\
l
\end{array}\right) \partial_{t^{l}}(u-v) \cdot\left[\left(\sum_{j=0}^{M-1} \sum_{r=0}^{k-l}\left(\begin{array}{c}
k-l \\
r
\end{array}\right) \partial_{t^{r}} u^{j} \cdot \partial_{t^{k-l-r}} v^{M-1-j}\right)\right] .
\end{aligned}
$$

In order to estimate the terms $\partial_{t^{r}} u^{j}$ and $\partial_{t^{k-l-r}} v^{M-1-j}$ we apply Faà di Bruno's formula

$$
\partial_{t^{r}}(f \circ g)=\sum \frac{r !}{k_{1} ! \ldots k_{r} !} 6\left(\partial_{t^{k_{1}+\cdots+k_{r}}} f \circ g\right) \prod_{i=1}^{r}\left(\frac{\partial_{t^{i}} g}{i !}\right)^{k_{i}}
$$

where the sum runs over all $r$-tuples of nonnegative integers $\left(k_{1}, \ldots, k_{r}\right)$ satisfying

$$
1 \cdot k_{1}+2 \cdot k_{2}+\cdots+r \cdot k_{r}=r .
$$

In particular, from (4.15) we see that $k_{r} \leq 1$, where $r=1, \ldots, k$. Therefore, the highest derivative $\partial_{t^{r}} u$ appears at most once. We apply the formula to $g=u$ and $f(x)=x^{j}$ and make use of the embeddings (2.3) and the pointwise multiplier results from Theorem 1 (i) for $k \leq \gamma_{m}-1$. (Note that the restriction ' $a>\frac{d}{p}$ ' for $d=3$ in Theorem 1 (i) is satisfied since in our situation we have $a+2 m \geq m>\frac{d}{2}$ from the assumptions $a \in[-m, m]$ and $m \geq 2$.) This yields

$$
\begin{aligned}
& \left\|\partial_{t^{r}} u^{j} \mid \mathcal{K}_{2, a+2 m\left(\gamma_{m}-k\right)}^{2 m\left(\gamma_{m}-k\right)}(D)\right\| \\
& \leq c_{r, j}\left\|\sum_{\substack{k_{1}+\cdots+k_{r} \leq j, 1 \cdot k_{1}+2 \cdot k_{2}+\cdots+r \cdot k_{r}=r}} u^{j-\left(k_{1}+\cdots+k_{r}\right)} \prod_{i=1}^{r}\left|\partial_{t^{i}} u\right|^{k_{i}} \mid \mathcal{K}_{2, a+2 m\left(\gamma_{m}-k\right)}^{2 m\left(\gamma_{m}-k\right)}(D)\right\| \\
& \lesssim \quad \sum_{k_{1}+\cdots+k_{r} \leq j,}\left\|u\left|\mathcal{K}_{2, a+2 m\left(\gamma_{m}-k\right)}^{2 m\left(\gamma_{m}-k^{\prime}\right)}(D)\left\|^{j-\left(k_{1}+\cdots+k_{r}\right)} \prod_{i=1}^{r}\right\| \partial_{t^{i}} u\right| \mathcal{K}_{2, a+2 m\left(\gamma_{m}-k\right)}^{2 m\left(\gamma_{m}-k\right)}(D)\right\|^{k_{i}} .
\end{aligned}
$$


For $k=\gamma_{m}$ we use Theorem 1(ii). (Note that in Theorem 1(ii) we require that ' $a-1 \geq$ $\frac{d}{p}-2$ ' with $d=3$ for the parameter. In our situation below $a-1$ has to be replaced by $a$, which leads to our restriction $a \geq \frac{d}{2}-2=-\frac{1}{2}$.) Similar as above we obtain

$$
\begin{aligned}
& \left\|\partial_{t^{r}} u^{j} \mid \mathcal{K}_{2, a}^{0}(D)\right\| \\
& \leq c_{r, j}\left\|\sum_{\substack{k_{1}+\cdots+k_{r} \leq j, 1 \cdot k_{1}+2 \cdot k_{2}+\cdots+r \cdot k_{r}=r}} u^{j-\left(k_{1}+\cdots+k_{r}\right)} \prod_{i=1}^{r}\left|\partial_{t^{i}} u\right|^{k_{i}} \mid \mathcal{K}_{2, a}^{0}(D)\right\| \\
& \lesssim \sum_{\substack{k_{1}+\cdots+k_{r} \leq j, 1 \cdot k_{1}+2 \cdot k_{2}+\cdots+r \cdot k_{r}=r}}\left\|u\left|\mathcal{K}_{2, a+2}^{2}(D)\left\|^{j-\left(k_{1}+\cdots+k_{r}\right)}\right\| \partial_{t^{r}} u\right| \mathcal{K}_{2, a}^{0}(D)\right\|^{k_{r}} \prod_{i=1}^{r-1}\left\|\partial_{t^{i}} u \mid \mathcal{K}_{2, a+2}^{2}(D)\right\|^{k_{i}} \\
& \lesssim \sum_{\substack{1 \cdot k_{1}+2 \cdot k_{2}+\cdots+k_{r} \leq j, k_{1}+k_{r}=r}}\left\|u \mid \mathcal{K}_{2, a+2 m \gamma_{m}}^{2 m \gamma_{m}}(D)\right\| \|^{j-\left(k_{1}+\cdots+k_{r}\right)} \\
& \left\|\partial_{t^{r}} u\left|\mathcal{K}_{2, a+2 m\left(\gamma_{m}-r\right)}^{2 m\left(\gamma_{m}-r\right)}(D)\left\|^{k_{r}} \prod_{i=1}^{r-1}\right\| \partial_{t^{i}} u\right| \mathcal{K}_{2, a+2 m\left(\gamma_{m}-i\right)}^{2 m\left(\gamma_{m}-i\right)}(D)\right\|^{k_{i}}
\end{aligned}
$$

Note that we require $\gamma_{m} \geq 1$ in the last step. We proceed similarly for $\partial_{t^{k-l-r}} v^{M-1-j}$. Now (4.13) together with (4.16) and (4.17) inserted in (4.12) together with Theorem 1 give

$$
\begin{aligned}
& \left\|\tilde{L}^{-1}\right\|\left\|u^{M}-v^{M} \mid \mathcal{D}_{1}\right\| \\
& \lesssim\left\|\tilde{L}^{-1}\right\| \sum_{k=0}^{\gamma_{m}}\left(\int_{0}^{T}\left\|\partial_{t^{k}}\left[(u-v) \sum_{j=0}^{M-1} u^{j} v^{M-1-j}\right] \mid \mathcal{K}_{2, a+2 m\left(\gamma_{m}-k\right)}^{2 m\left(\gamma_{m}-k\right)}(D)\right\|^{2} \mathrm{~d} t\right)^{1 / 2} \\
& \lesssim\left\|\tilde{L}^{-1}\right\| \sum_{k=0}^{\gamma_{m}} \sum_{l=0}^{k} \sum_{j=0}^{M-1} \sum_{r=0}^{k-l}\left(\int_{0}^{T}\left\|\partial_{t}(u-v) \mid \mathcal{K}_{2, a+2 m\left(\gamma_{m}-k\right)}^{2 m\left(\gamma_{m}-k\right)}(D)\right\|^{2}\right. \\
& \left.\left\|\partial_{t^{r}} u^{j}\left|\mathcal{K}_{2, a+2 m\left(\gamma_{m}-k\right)}^{2 m\left(\gamma_{m}-k\right)}(D)\left\|^{2}\right\| \partial_{t^{k-l-r}} v^{M-1-j}\right| \mathcal{K}_{2, a+2 m\left(\gamma_{m}-k\right)}^{2 m\left(\gamma_{m}-k\right)}(D)\right\|^{2} \mathrm{~d} t\right)^{1 / 2} \\
& \lesssim\left\|\tilde{L}^{-1}\right\| \sum_{k=0}^{\gamma_{m}} \sum_{l=0}^{k} \sum_{j=0}^{M-1} \sum_{r=0}^{k-l}\left(\int_{0}^{T}\left\|\partial_{t}(u-v) \mid \mathcal{K}_{2, a+2 m\left(\gamma_{m}-k\right)}^{2 m\left(\gamma_{m}-k\right)}(D)\right\|^{2}\right. \\
& \sum_{\kappa_{1}+\cdots+\kappa_{r} \leq j,}\left\|u\left|\mathcal{K}_{2, a+2 m\left(\gamma_{m}-k\right)}^{2 m\left(\gamma_{m}-k\right)}(D)\left\|^{2\left(j-\left(\kappa_{1}+\cdots+\kappa_{r}\right)\right)} \prod_{i=0}^{r}\right\| \partial_{t^{i}} u\right| \mathcal{K}_{2, a+2 m\left(\gamma_{m}-i\right)}^{2 m\left(\gamma_{m}-i\right)}(D)\right\|^{2 \kappa_{i}} \\
& \kappa_{1}+2 \kappa_{2}+\cdots+r \kappa_{r}=r
\end{aligned}
$$




$$
\begin{aligned}
& \sum_{\kappa_{1}+\cdots+\kappa_{k-l-r} \leq M-1-j,}\left\|v \mid \mathcal{K}_{2, a+2 m\left(\gamma_{m}-k\right)}^{2 m\left(\gamma_{m}-k\right)}(D)\right\|^{2\left(M-1-j-\left(\kappa_{1}+\cdots+\kappa_{k-l-r}\right)\right)} \\
& \kappa_{1}+2 \kappa_{2}+\cdots+(k-l-r) \kappa_{k-l-r}=k-l-r \\
& \left.\prod_{i=0}^{k-l-r}\left\|\partial_{t^{i}} v \mid \mathcal{K}_{2, a+2 m\left(\gamma_{m}-i\right)}^{2 m\left(\gamma_{m}-i\right)}(D)\right\|^{2 \kappa_{i}} \mathrm{~d} t\right)^{1 / 2} \\
& \lesssim\left\|\tilde{L}^{-1}\right\| \sum_{k=0}^{\gamma_{m}} M\left(\int_{0}^{T}\left\|\partial_{t^{k}}(u-v) \mid \mathcal{K}_{2, a+2 m\left(\gamma_{m}-k\right)}^{2 m\left(\gamma_{m}-k\right)}(D)\right\|^{2}\right. \\
& \sum_{\kappa_{1}^{\prime}+\cdots+\kappa_{k}^{\prime} \leq \min \{M-1, k\},} \max _{w \in\{u, v\}}\left\|w \mid \mathcal{K}_{2, a+2 m\left(\gamma_{m}-k\right)}^{2 m\left(\gamma_{m}-k\right)}(D)\right\|^{2\left(M-1-\left(\kappa_{1}^{\prime}+\cdots+\kappa_{k}^{\prime}\right)\right)} \\
& \kappa_{k}^{\prime} \leq 1 \\
& \left.\prod_{i=0}^{k} \max \left\{\left\|\partial_{t^{i}} u\left|\mathcal{K}_{2, a+2 m\left(\gamma_{m}-i\right)}^{2 m\left(\gamma_{m}-i\right)}(D)\|,\| \partial_{t^{i}} v\right| \mathcal{K}_{2, a+2 m\left(\gamma_{m}-i\right)}^{2 m\left(\gamma_{m}-i\right)}(D)\right\|, 1\right\}^{4 \kappa_{i}^{\prime}} \mathrm{d} t\right)^{1 / 2} \\
& \lesssim M\left\|\tilde{L}^{-1}\right\| \cdot\left\|u-v \mid \bigcap_{k=0}^{\gamma_{m}+1} H^{k}\left([0, T], \mathcal{K}_{2, a+2 m\left(\gamma_{m}-(k-1)\right)}^{2 m\left(\gamma_{m}-(k-1)\right)}(D)\right)\right\| . \\
& \max _{w \in\{u, v\}} \max _{l=0, \ldots, \gamma_{m}} \max \left(\left\|\partial_{t^{l}} w \mid L_{\infty}\left([0, T], \mathcal{K}_{2, a+2 m\left(\gamma_{m}-l\right)}^{2 m\left(\gamma_{m}-l\right)}(D)\right)\right\|, 1\right)^{2(M-1)} .
\end{aligned}
$$

We give some explanations concerning the estimate above. In (4.18) the term with $k=\gamma_{m}$ requires some special care since we have to apply Theorem 1 (ii). In this case, we calculate

$$
\begin{aligned}
& \left\|\partial_{\gamma_{m}}\left[(u-v)\left(\sum_{j=0}^{M-1} u^{j} v^{M-1-j}\right)\right] \mid \mathcal{K}_{2, a}^{0}(D)\right\| \\
& \lesssim\left\|\partial_{\gamma_{m}}(u-v)\left|\mathcal{K}_{2, a}^{0}(D)\left\|\sum_{j=0}^{M-1}\right\| u^{j} v^{M-1-j}\right| \mathcal{K}_{2, a+2}^{2}(D)\right\| \\
& +\left\|u-v\left|\mathcal{K}_{2, a+2}^{2}(D)\left\|\sum_{j=0}^{M-1} \sum_{r=0}^{\gamma_{m}}\right\|\left(\partial_{t} r u^{j}\right)\left(\partial_{t \gamma_{m}-r} v^{M-1-j}\right)\right| \mathcal{K}_{2, a}^{0}(D)\right\| \\
& \quad+\left\|\sum_{r=1}^{\gamma_{m}-1}\left(\begin{array}{c}
\gamma_{m} \\
r
\end{array}\right) \partial_{r}(u-v) \partial_{\gamma_{m}-r}\left(\sum_{j=0}^{M-1} \cdots\right) \mid \mathcal{K}_{2, a}^{0}(D)\right\| .
\end{aligned}
$$

The lower order derivatives in the last line cause no problems since we can (again) apply Theorem 1(i) as before. The term $\left\|u^{j} v^{M-1-j} \mid \mathcal{K}_{2, a+2}^{2}(D)\right\|$ can now be further estimated with the help of Theorem 1(i). For the term $\sum_{r=0}^{\gamma_{m}}$ 
$\left\|\left(\partial_{t} r u^{j}\right)\left(\partial_{t \gamma_{m}-r} v^{M-1-j}\right) \mid \mathcal{K}_{2, a}^{0}(D)\right\|$ we again use Theorem 1(ii), then proceed as in (4.17) and see that the resulting estimate yields (4.18).

Moreover, in (4.19) we use the fact that in the step before we have two sums with $\kappa_{1}+\cdots+\kappa_{r} \leq j$ and $\kappa_{1}+\cdots+\kappa_{k-l-r} \leq M-1-j$, i.e., we have $k-l$ different $\kappa_{i}$ 's which leads to at most $k$ different $\kappa_{i}$ 's if $l=0$. We allow all combinations of $\kappa_{i}$ 's and redefine the $\kappa_{i}$ 's in the second sum leading to $\kappa_{1}^{\prime}, \ldots, \kappa_{k}^{\prime}$ with $\kappa_{1}^{\prime}+\cdots+\kappa_{k}^{\prime} \leq M-1$ and replace the old conditions $\kappa_{1}+2 \kappa_{2}+r \kappa_{r} \leq r$ and $\kappa_{1}+2 \kappa_{2}+(k-l-r) \kappa_{k-l-r} \leq k-l-r$ by the weaker ones $\kappa_{1}^{\prime}+\cdots+\kappa_{k}^{\prime} \leq k$ and $\kappa_{k}^{\prime} \leq 1$. This causes no problems since the other terms appearing in this step do not depend on $\kappa_{i}$ apart from the product term. There, the fact that some of the old $\kappa_{i}$ 's from both sums might coincide is reflected in the new exponent $4 \kappa_{i}^{\prime}$. From Theorem 1 we conclude that

$$
\begin{aligned}
u, v \in S & \hookrightarrow \bigcap_{k=0}^{\gamma_{m}+1} H^{k}\left([0, T], \mathcal{K}_{2, a+2 m\left(\gamma_{m}-(k-1)\right)}^{2 m\left(\gamma_{m}-(k-1)\right)}(D)\right) \\
& \hookrightarrow \bigcap_{k=1}^{\gamma_{m}+1} \mathcal{C}^{k-1, \frac{1}{2}}\left([0, T], \mathcal{K}_{2, a+2 m\left(\gamma_{m}-(k-1)\right)}^{\left.2 m\left(\gamma_{m}-(k)\right)\right)}\right. \\
& \hookrightarrow \bigcap_{k=1}^{\gamma_{m}+1} C^{k-1}\left([0, T], \mathcal{K}_{2, a+2 m\left(\gamma_{m}-(k-1)\right)}^{2 m\left(\gamma_{m}-(k-1)\right)}(D)\right)=\bigcap_{l=0}^{\gamma_{m}} C^{l}\left([0, T], \mathcal{K}_{2, a+2 m\left(\gamma_{m}-l\right)}^{2 m\left(\gamma_{m}-l\right)}(D)\right),
\end{aligned}
$$

hence, the term involving the maxima, $\max _{w \in\{u, v\}} \max _{l=0, \ldots, \gamma_{m}} \max (\ldots)^{M-1}$ in (4.20) is bounded by $\max \left(R+\left\|\tilde{L}^{-1} f \mid S\right\|, 1\right)^{M-1}$. Moreover, since $u$ and $v$ are taken from $B_{R}\left(\tilde{L}^{-1} f\right)$ in $S=S_{1} \cap S_{2}$, we obtain from (4.20),

$$
\begin{aligned}
\left\|\tilde{L}^{-1}\right\| & \left\|u^{M}-v^{M} \mid \mathcal{D}_{1}\right\| \\
& \leq c_{0}\left\|\tilde{L}^{-1}\right\| M \max \left(R+\left\|\tilde{L}^{-1} f \mid S\right\|, 1\right)^{2(M-1)}\|u-v \mid S\| \\
& \leq c_{2}\left\|\tilde{L}^{-1}\right\| M \max \left(R+\left\|\tilde{L}^{-1}\right\| \cdot\|f \mid \mathcal{D}\|, 1\right)^{2(M-1)}\|u-v \mid S\| \\
& =c_{2}\left\|\tilde{L}^{-1}\right\| M \max \left(R+\left\|\tilde{L}^{-1}\right\| \eta, 1\right)^{2(M-1)}\|u-v \mid S\|,
\end{aligned}
$$

where we put $\eta:=\|f \mid \mathcal{D}\|$ in the last line, $c_{0}$ denotes the constant resulting from (4.16) and (4.20) and $c_{2}=c_{0} c_{1}$ with $c_{1}$ being the constant from the estimates in Theorem 5.

We now turn our attention towards the second term $\left\|\tilde{L}^{-1}\right\|\left\|u^{M}-v^{M} \mid \mathcal{D}_{2}\right\|$ in (4.12) and calculate

$$
\begin{aligned}
& \left\|\tilde{L}^{-1}\right\|\left\|\left(u^{M}-v^{M}\right) \mid \mathcal{D}_{2}\right\| \\
& =\left\|\tilde{L}^{-1}\right\|\left\|(u-v) \sum_{j=0}^{M-1} u^{j} v^{M-1-j} \mid H^{\gamma_{m}+1}\left([0, T], L_{2}(D)\right)\right\| \\
& =\left\|\tilde{L}^{-1}\right\| \sum_{k=0}^{\gamma_{m}+1}\left\|\partial_{t^{k}}\left[(u-v) \sum_{j=0}^{M-1} u^{j} v^{M-1-j}\right] \mid L_{2}\left(D_{T}\right)\right\| \\
& =\left\|\tilde{L}^{-1}\right\| \sum_{k=0}^{\gamma_{m}+1}\left\|\sum_{l=0}^{k}\left(\begin{array}{c}
k \\
l
\end{array}\right) \partial_{t^{l}}(u-v) \cdot\left[\left(\sum_{j=0}^{M-1} \sum_{r=0}^{k-l}\left(\begin{array}{c}
k-l \\
r
\end{array}\right) \partial_{t^{r}} u^{j} \cdot \partial_{t^{k-l-r}} v^{M-1-j}\right)\right] \mid L_{2}\left(D_{T}\right)\right\|
\end{aligned}
$$




$$
\lesssim\left\|\tilde{L}^{-1}\right\| \sum_{k=0}^{\gamma_{m}+1}\left\|\sum_{l=0}^{k}\left|\partial_{t^{l}}(u-v)\right| \cdot\left[\left(\sum_{j=0}^{M-1} \sum_{r=0}^{k-l}\left|\partial_{t^{r}} u^{j} \cdot \partial_{t^{k-l-r}} v^{M-1-j}\right|\right)\right] \mid L_{2}\left(D_{T}\right)\right\|,
$$

where we used Leibniz's formula twice as in (4.13) in the second but last line. Again Faà di Bruno's formula, cf. (4.14), is applied in order to estimate the derivatives in (4.22). We use a special case of the multiplier result from [39, Sect. 4.6.1, Thm. 1(i)], which tells us that for $m>\frac{3}{2}$ we have

$$
\left\|u v\left|L_{2}\|\lesssim\| u\right| H^{m}\right\| \cdot\left\|v \mid L_{2}\right\|
$$

(we remark that this is exactly the point where our assumption $m \geq 2$ comes into play). With this we obtain

$$
\begin{aligned}
\left\|\partial_{t^{r}} u^{j} \mid L_{2}(D)\right\| & \leq c_{r, j}\left\|\sum_{k_{1}+\cdots+k_{r} \leq j} u^{j-\left(k_{1}+\cdots+k_{r}\right)} \prod_{i=1}^{r}\left|\partial_{t^{i}} u\right|^{k_{i}} \mid L_{2}(D)\right\| \\
& \lesssim \sum_{k_{1}+\cdots+k_{r} \leq j}\left\|u\left|H^{m}(D)\left\|^{j-\left(k_{1}+\cdots+k_{r}\right)} \prod_{i=1}^{r-1}\right\| \partial_{t^{i}} u\right| H^{m}(D)\right\|^{k_{i}}\left\|\partial_{t^{r}} u \mid L_{2}(D)\right\|^{k_{r}}
\end{aligned}
$$

Similar for $\partial_{t^{k-l-r}} v^{M-1-j}$. As before, from (4.15) we observe $k_{r} \leq 1$, therefore the highest derivative $u^{(r)}$ appears at most once. Note that since $H^{m}(D)$ is a multiplication algebra for $m>\frac{d}{2}$, we get (4.24) with $L_{2}(D)$ replaced by $H^{m}(D)$ as well. Now (4.23) and (4.24) inserted in (4.22) give

$$
\begin{aligned}
& \left\|\tilde{L}^{-1}\right\|\left\|u^{M}-v^{M} \mid \mathcal{D}_{2}\right\| \\
& =\left\|\tilde{L}^{-1}\right\| \sum_{k=0}^{\gamma_{m}+1}\left(\int_{0}^{T}\left\|\partial_{t^{k}}(u-v) \sum_{j=0}^{M-1} u^{j} v^{M-1-j} \mid L_{2}(D)\right\|^{2} \mathrm{~d} t\right)^{1 / 2} \\
& \lesssim\left\|\tilde{L}^{-1}\right\| \sum_{k=0}^{\gamma_{m}+1} \sum_{l=0}^{k}\left(\int_{0}^{T}\left\|\partial_{t^{l}}(u-v) \mid H^{m}(D)\right\|^{2}\right. \\
& \left.\sum_{j=0}^{M-1} \sum_{r=0}^{k-l}\left\|\partial_{t^{r}} u^{j} \cdot \partial_{t^{k-l-r}} v^{M-1-j} \mid L_{2}(D)\right\|^{2} \mathrm{~d} t\right)^{1 / 2} \\
& \vdots\left\|\tilde{L}^{-1}\right\| \sum_{k=0}^{\gamma_{m}+1} \sum_{l=0}^{k}\left(\int _ { 0 } ^ { T } \left\{\left\|\partial_{t^{l}}(u-v) \mid H^{m}(D)\right\|^{2}\right.\right. \\
& \sum_{j=0}^{M-1} \sum_{\left(k-l-r \neq \gamma_{m}+1\right) \wedge\left(r \neq \gamma_{m}+1\right)} \sum_{k-l}\left\|\partial_{t^{r}} u^{j}\left|H^{m}(D)\left\|^{2}\right\| \partial_{t^{k-l-r}} v^{M-1-j}\right| H^{m}(D)\right\|^{2} \\
& +\left\|u-v\left|H^{m}(D)\left\|^{2}\right\| \partial_{t^{\prime} \gamma_{m}+1} u^{j}\right| L_{2}(D)\right\|^{2}\left\|v^{M-1-j} \mid H^{m}(D)\right\|^{2}
\end{aligned}
$$




$$
\begin{aligned}
& \left.\left.+\left\|u-v\left|H^{m}(D)\left\|^{2}\right\| u^{j}\right| H^{m}(D)\right\|^{2}\left\|\partial_{t \gamma_{m}+1} v^{M-1-j} \mid L_{2}(D)\right\|^{2}\right\} \mathrm{~d} t\right)^{1 / 2} \\
& \lesssim\left\|\tilde{L}^{-1}\right\| \sum_{k=0}^{\gamma_{m}+1} \sum_{l=0}^{k}\left(\int_{0}^{T}\left\|\partial_{t^{l}}(u-v) \mid H^{m}(D)\right\|^{2}\right. \\
& \sum_{j=0}^{M-1} \sum_{r=0}^{k-l} \sum_{\substack{\kappa_{1}+\cdots+\kappa_{r} \leq j, \kappa_{1}+2 \kappa_{2}+\cdots+\kappa_{r} \leq r}}\left\|u \mid H^{m}(D)\right\|^{2\left(j-\left(\kappa_{1}+\cdots+\kappa_{r}\right)\right)} \\
& \left\{\begin{array}{ll}
\left\|\partial_{t^{r}} u\left|L_{2}(D)\left\|^{2 \kappa_{r}} \prod_{i=1}^{r-1}\right\| \partial_{t^{i}} u\right| H^{m}(D)\right\|^{2 \kappa_{i}}, & r=\gamma_{m}+1 \\
\prod_{i=1}^{r}\left\|\partial_{t^{i}} u \mid H^{m}(D)\right\|^{2 \kappa_{i}}, & r \neq \gamma_{m}+1
\end{array}\right\} \\
& \sum\left\|v \mid H^{m}(D)\right\|^{2\left(M-1-j-\left(\kappa_{1}+\cdots+\kappa_{k-l-r}\right)\right)} \\
& \kappa_{1}+\cdots+\kappa_{k-l-r} \leq M-1-j, \\
& \kappa_{1}+2 \kappa_{2}+\cdots+(k-l-r) \kappa_{k-l-r} \leq k-l-r \\
& \left.\left\{\begin{array}{ll}
\left\|\partial_{t^{r}} v\left|L_{2}(D)\left\|^{2 \kappa_{r}} \prod_{i=1}^{k-l-r-1}\right\| \partial_{t^{i}} v\right| H^{m}(D)\right\|^{2 \kappa_{i}}, & k-l-r=\gamma_{m}+1, \\
\prod_{i=1}^{l-k-r}\left\|\partial_{t^{i}} v \mid H^{m}(D)\right\|^{2 \kappa_{i}}, & k-l-r \neq \gamma_{m}+1
\end{array}\right\} \mathrm{d} t\right)^{1 / 2} \\
& \lesssim\left\|\tilde{L}^{-1}\right\| \sum_{k=0}^{\gamma_{m}+1}\left(\int_{0}^{T}\left\|\partial_{t^{k}}(u-v) \mid H^{m}(D)\right\|^{2}\right. \\
& M \sum_{\kappa_{1}^{\prime}+\cdots+\kappa_{k}^{\prime} \leq \min \{M-1, k\}} \max _{w \in\{u, v\}}\left\|w \mid H^{m}(D)\right\|^{2\left(M-1-\left(\kappa_{1}^{\prime}+\cdots+\kappa_{k}^{\prime}\right)\right)} \\
& \left.\left\{\begin{array}{ll}
\max \left(\left\|\partial_{t^{k}} w\left|L_{2}(D)\left\|^{4 \kappa_{k}^{\prime}} \prod_{i=1}^{k-1}\right\| \partial_{t^{i}} w\right| H^{m}(D)\right\|^{4 \kappa_{i}^{\prime}}, 1\right), & k=\gamma_{m}+1, \\
\max \left(\prod_{i=1}^{k}\left\|\partial_{t^{i}} w \mid H^{m}(D)\right\|^{4 \kappa_{i}^{\prime}}, 1\right), & k \neq \gamma_{m}+1
\end{array}\right\} \mathrm{d} t\right)^{1 / 2} \\
& \lesssim\left\|\tilde{L}^{-1}\right\| M\left\|u-v \mid H^{\gamma_{m}+1}\left([0, T], H^{m}(D)\right)\right\|^{2} \max _{w \in\{u, v\}} \max _{i=0, \ldots, \gamma_{m}} \max \\
& \left(\left\|\partial_{t^{i}} w\left|L_{\infty}\left([0, T], H^{m}(D)\right)\|,\| \partial_{t^{\gamma m}+1} w\right| L_{\infty}\left([0, T], L_{2}(D)\right)\right\|, 1\right)^{2(M-1)} .
\end{aligned}
$$

Similar to (4.20) in the calculations above the term $k=\gamma_{m}+1$ required some special care. For the redefinition of the $\kappa_{i}$ 's in the second but last line in (4.25) we refer to the explanations given after (4.20). From Theorem 1 we see that

$$
\begin{aligned}
u, v \in S & \hookrightarrow H^{\gamma_{m}+1}\left([0, T], \stackrel{\circ}{H}^{m}(D)\right) \cap H^{\gamma_{m}+2}\left([0, T], L_{2}(D)\right) \\
& \hookrightarrow \mathcal{C}^{\gamma_{m}}, \frac{1}{2}\left([0, T], \stackrel{\circ}{H}^{m}(D)\right) \cap \mathcal{C}^{\gamma_{m}+1, \frac{1}{2}}\left([0, T], L_{2}(D)\right) \\
& \hookrightarrow C^{\gamma_{m}}\left([0, T], \stackrel{\circ}{H}^{m}(D)\right) \cap C^{\gamma_{m}+1}\left([0, T], L_{2}(D)\right),
\end{aligned}
$$

hence the term $\max _{w \in\{u, v\}} \max _{m=0, \ldots, l} \max (\ldots)^{M-1}$ in (4.25) is bounded. Moreover, since $u$ and $v$ are taken from $B_{R}\left(\tilde{L}^{-1} f\right)$ in $S_{2}=H^{m, \gamma_{m}+2 *}\left(D_{T}\right)=$ $H^{\gamma_{m}+1}\left([0, T], \stackrel{\circ}{H}^{m}(D)\right) \cap H^{\gamma_{m}+2}\left([0, T], H^{-m}(D)\right)$, as in (4.21) we obtain from (4.25) 
and (4.26),

$$
\left\|\tilde{L}^{-1}\right\|\left\|u^{M}-v^{M}\left|\mathcal{D}_{2}\left\|\leq c_{3}\right\| \tilde{L}^{-1}\left\|M \max \left(R+\left\|\tilde{L}^{-1}\right\| \eta, 1\right)^{2(M-1)} \cdot\right\| u-v\right| S\right\|,
$$

where we put $\eta:=\|f \mid \mathcal{D}\|$ and $c_{3}$ denotes the constant arising from our estimates (4.25) and (4.26) above. Now (4.12) together with (4.21) and (4.27) yields

$$
\begin{aligned}
\left\|\tilde{L}^{-1}\left(u^{M}-v^{M}\right) \mid S\right\| & \leq\left\|\tilde{L}^{-1}\right\|\left\|\left(u^{M}-v^{M}\right) \mid \tilde{\mathcal{D}}\right\| \\
& \leq c\left\|\tilde{L}^{-1}\right\| M \max \left(R+\left\|\tilde{L}^{-1}\right\| \eta, 1\right)^{M-1}\|u-v \mid S\|,
\end{aligned}
$$

where $c=c_{2}+c_{3}$. For $\tilde{L}^{-1} \circ N$ to be a contraction, we therefore require

$$
c \varepsilon\left\|\tilde{L}^{-1}\right\| M \max \left(R+\left\|\tilde{L}^{-1}\right\| \eta, 1\right)^{2(M-1)}<1,
$$

cf. (4.11). In case of $\max \left(R+\left\|\tilde{L}^{-1}\right\| \eta, 1\right)=1$ this leads to

$$
\left\|\tilde{L}^{-1}\right\|<\frac{1}{c \varepsilon M} .
$$

On the other hand, if $\max \left(R+\left\|\tilde{L}^{-1}\right\| \eta, 1\right)=R+\left\|\tilde{L}^{-1}\right\| \eta$, we choose $R=\left(r_{0}-\right.$ 1) $\eta\left\|\tilde{L}^{-1}\right\|$, which gives rise to the condition

$$
\begin{aligned}
& c \varepsilon\left\|\tilde{L}^{-1}\right\| M\left(r_{0}\left\|\tilde{L}^{-1}\right\| \eta\right)^{2(M-1)}<1, \text { i.e., } \eta^{2(M-1)}\left\|\tilde{L}^{-1}\right\|^{2 M-1} \\
& <\frac{1}{c \varepsilon M}\left(\frac{1}{r_{0}}\right)^{2(M-1)} .
\end{aligned}
$$

Step 2: The calculations in Step 1 show that $u^{M} \in \widetilde{\mathcal{D}}$ : The fact that $u^{M} \in \mathcal{D}_{1} \cap \mathcal{D}_{2}$ follows from the estimate (4.28). In particular, taking $v=0$ in (4.28) we get an estimate from above for $\left\|u^{M} \mid \widetilde{\mathcal{D}}\right\|$. The upper bound depends on $\|u \mid S\|$ and several constants which depend on $u$ but are finite whenever we have $u \in S$, see also (4.20) and (4.25). The dependence on $R$ in (4.28) comes from the fact that we choose $u \in$ $B_{R}\left(\tilde{L}^{-1} f\right)$ in $S$ there. However, the same argument can also be applied to an arbitrary $u \in S$; this would result in a different constant $\tilde{c}$. In order to have $u^{M} \in \widetilde{\mathcal{D}}$, we still need to show that $\operatorname{Tr}\left(\partial_{t^{k}} u^{M}\right)=0, k=0, \ldots, \gamma_{m}$. This follows from the same arguments as in [22, Thm. 4.10]: Since $u \in S \hookrightarrow H^{\gamma_{m}+2}\left([0, T], H^{-m}(D)\right) \hookrightarrow$ $C^{\gamma_{m}+1}\left([0, T], H^{-m}(D)\right)$ we see that the trace operator $\operatorname{Tr}\left(\partial_{t^{k}} u\right):=\left(\partial_{t^{k}} u\right)(0, \cdot)$ is well defined for $k=0, \ldots, \gamma_{m}+1$. Using the initial assumption $u(0, \cdot)=0$ in Problem 2 , by density arguments $\left(C^{\infty}\left(D_{T}\right)\right.$ is dense in $\left.S\right)$ and induction we deduce that $\left(\partial_{t^{k}} u\right)(0, \cdot)=0$ for all $k=0, \ldots, \gamma_{m}+1$. Moreover, since by Theorem 1

$$
u^{M} \in \mathcal{D}_{1} \cap \mathcal{D}_{2} \hookrightarrow H^{\gamma_{m}+1}\left([0, T], L_{2}(D)\right) \hookrightarrow C^{\gamma_{m}}\left([0, T], L_{2}(D)\right),
$$

we see that the trace operator $\operatorname{Tr}\left(\partial_{t^{k}} u^{M}\right):=\left(\partial_{t^{k}} u^{M}\right)(0, \cdot)$ is well defined for $k=$ $0, \ldots, \gamma_{m}$. By (4.24) below the term $\left\|\left(\partial_{t^{k}} u^{M}\right)(0, \cdot) \mid L_{2}(D)\right\|$ is estimated from above 
by powers of $\left\|\left(\partial_{t} l u\right)(0, \cdot) \mid H^{m}(D)\right\|, l=0, \ldots, k$. Since all these terms are equal to zero, this shows that $u^{M} \in \widetilde{\mathcal{D}}$.

Step 3: The next step is to show that $\left(\tilde{L}^{-1} \circ N\right)\left(B_{R}\left(\tilde{L}^{-1} f\right)\right) \subset B_{R}\left(\tilde{L}^{-1} f\right)$ in $S$. Since $\left(\tilde{L}^{-1} \circ N\right)(0)=\tilde{L}^{-1}\left(f-\varepsilon 0^{M}\right)=\tilde{L}^{-1} f$, we only need to apply the above estimate (4.28) with $v=0$. This gives

$$
\begin{gathered}
\varepsilon\left\|\tilde{L}^{-1} u^{M} \mid S\right\| \leq c \varepsilon\left\|\tilde{L}^{-1}\right\| M \max \left(R+\left\|\tilde{L}^{-1}\right\| \eta, 1\right)^{2(M-1)}\left(R+\left\|\tilde{L}^{-1}\right\| \eta\right) \\
\stackrel{!}{\leq} R=\left(r_{0}-1\right) \eta\left\|\tilde{L}^{-1}\right\|,
\end{gathered}
$$

which, in case that $\max \left(R+\left\|\tilde{L}^{-1}\right\| \eta, 1\right)=1$, leads to

$$
\left\|\tilde{L}^{-1}\right\|<\frac{r_{0}-1}{r_{0}}\left(\frac{1}{c \varepsilon M}\right),
$$

whereas for $\max \left(R+\left\|\tilde{L}^{-1}\right\| \eta, 1\right)=R+\left\|\tilde{L}^{-1}\right\| \eta$ we get

$$
\eta^{2(M-1)}\left\|\tilde{L}^{-1}\right\|^{2 M-1} \leq \frac{1}{c \varepsilon M}\left(r_{0}-1\right)\left(\frac{1}{r_{0}}\right)^{2 M-1} .
$$

We see that condition (4.31) implies (4.29). Furthermore, since

$$
\left(r_{0}-1\right)\left(\frac{1}{r_{0}}\right)^{2 M-1}=\frac{r_{0}-1}{r_{0}}\left(\frac{1}{r_{0}}\right)^{2(M-1)}<\left(\frac{1}{r_{0}}\right)^{2(M-1)},
$$

also condition (4.32) implies (4.30). Thus, by applying Banach's fixed point theorem in a sufficiently small ball around the solution of the corresponding linear problem, we obtain a unique solution of Problem 2.

Remark 9 The restriction $m \geq 2$ in Theorem 7 comes from the fact that we require $s_{2}=m>\frac{d}{2}=\frac{3}{2}$ in (4.23). This assumption can probably be weakened, since we expect the solution to satisfy $u \in L_{2}\left([0, T], H^{s}(D)\right)$ for all $s<\frac{3}{2}$, see also Remark 11 and the explanations given there.

Moreover, the restriction $a \geq-\frac{1}{2}$ in Theorem 7 comes from Theorem 1(ii) that we applied. Together with the restriction $a \in[-m, m]$ we are looking for $a \in\left[-\frac{1}{2}, m\right]$ if the domain is a corner domain, e.g., a smooth cone $K \subset \mathbb{R}^{3}$ (subject to some truncation). For polyhedral cones with edges $M_{k}, k=1, \ldots, l$, we furthermore require $-\delta_{-}^{(k)}<a+2 m\left(\gamma_{m}-i\right)+m<\delta_{+}^{(k)}$ for $i=0, \ldots, \gamma_{m}$ from Theorem 5.

\section{Regularity Results in Besov Spaces}

With all preliminary work, in this section we finally come to the presentation of the regularity results in Besov spaces for Problems 1 and 2. For this purpose, we rely on the results from Sect. 4 on regularity in Sobolev and Kondratiev spaces for the 
respective problems and combine these with the embeddings of Kondratiev spaces into Besov spaces. It turns out that in all cases studied the Besov regularity is higher than the Sobolev regularity. This indicates that adaptivity pays off when solving these problems numerically.

The Sobolev regularity we are working with (e.g., see Theorem 3 for Problem 1) canonically comes out from the variational formulation of the problem, i.e., we have spatial Sobolev regularity $m$ if the corresponding differential operator is of order $2 m$. We give an outlook on how our results could be improved by using regularity results in fractional Sobolev spaces instead. It is planned to do further investigations in this direction in the future.

Moreover, we discuss the role of the weight parameter $a$ appearing in our Kondratiev spaces to some extent.

\subsection{Besov Regularity of Problem I}

A combination of Theorem 5 (Kondratiev regularity A) and the embedding in Theorem 2 yields the following Besov regularity of Problem 1.

Theorem 8 (Parabolic Besov regularity A) Let D be a bounded polyhedral domain in $\mathbb{R}^{3}$. Let $\gamma \in \mathbb{N}$ with $\gamma \geq 2 m$ and put $\gamma_{m}:=\left[\frac{\gamma-1}{2 m}\right]$. Furthermore, let $a \in \mathbb{R}$ with $a \in[-m, m]$. Assume that the right-hand side $f$ of Problem 1 satisfies

(i) $\partial_{t^{k}} f \in L_{2}\left(D_{T}\right) \cap L_{2}\left([0, T], \mathcal{K}_{2, a+2 m\left(\gamma_{m}-k\right)}^{2 m\left(\gamma_{m}-k\right)}(D)\right), k=0, \ldots, \gamma_{m} ; \quad \partial_{t \gamma_{m}+1} f \in$ $L_{2}\left(D_{T}\right)$.

(ii) $\partial_{t^{k}} f(x, 0)=0, \quad k=0,1, \ldots, \gamma_{m}$.

Furthermore, let Assumption 1 hold for weight parameters $b=a+2 m\left(\gamma_{m}-i\right)$, where $i=0, \ldots, \gamma_{m}$, and $b^{\prime}=-m$. Then for the weak solution $u \in H^{m, \gamma_{m}+2 *}\left(D_{T}\right)$ of Problem 1, we have

$$
u \in L_{2}\left([0, T], B_{\tau, \infty}^{\alpha}(D)\right) \quad \text { for all } 0<\alpha<\min \left(\gamma, \frac{3}{\delta} m\right),
$$

where $\frac{1}{2}<\frac{1}{\tau}<\frac{\alpha}{d}+\frac{1}{2}$ and $\delta$ denotes the dimension of the singular set of $D$. In particular, for any $\alpha$ satisfying (5.1) and $\tau$ as above, we have the a priori estimate

$$
\begin{aligned}
& \left\|u\left|L_{2}\left([0, T], B_{\tau, \infty}^{\alpha}(D)\right)\left\|\lesssim \sum_{k=0}^{\gamma_{m}}\right\| \partial_{t^{k}} f\right| L_{2}\left([0, T], \mathcal{K}_{2, a+2 m\left(\gamma_{m}-k\right)}^{2 m\left(\gamma_{m}-k\right)}(D)\right)\right\| \\
& \quad+\sum_{k=0}^{\gamma_{m}+1}\left\|\partial_{t^{k}} f \mid L_{2}\left(D_{T}\right)\right\|
\end{aligned}
$$

Proof According to Theorem 5 by our assumptions we know $u \in L_{2}([0, T]$, $\mathcal{K}_{2, a+2 m\left(\gamma_{m}+1\right)}^{2 m\left(\gamma_{m}+1\right)}(D)$. Together with Theorem 2 (choosing $k=0$ ) we obtain

$$
u \in L_{2}\left([0, T], \mathcal{K}_{2, a+2 m\left(\gamma_{m}+1\right)}^{2 m\left(\gamma_{m}+1\right)}(D)\right) \cap H^{m, \gamma_{m}+2 *}\left(D_{T}\right)
$$




$$
\begin{aligned}
& \hookrightarrow L_{2}\left([0, T], \mathcal{K}_{2, a+2 m\left(\gamma_{m}+1\right)}^{2 m\left(\gamma_{m}+1\right)}(D)\right) \cap L_{2}\left([0, T], H^{m}(D)\right) \\
& \hookrightarrow L_{2}\left([0, T], \mathcal{K}_{2, a+2 m\left(\gamma_{m}+1\right)}^{2 m\left(\gamma_{m}+1\right)}(D)\right) \cap L_{2}\left([0, T], B_{2, \infty}^{m}(D)\right) \\
& \hookrightarrow L_{2}\left([0, T], \mathcal{K}_{2, a+2 m\left(\gamma_{m}+1\right)}^{\alpha}(D) \cap B_{2, \infty}^{m}(D)\right) \hookrightarrow L_{2}\left([0, T], B_{\tau, \infty}^{\alpha}(D)\right),
\end{aligned}
$$

where in the third step we use the fact that $2 m\left(\gamma_{m}+1\right) \geq 2 m\left(\frac{\gamma}{2 m}-1+1\right)=$ $\gamma$ and choose $\alpha \leq \gamma$. Moreover, the condition on $a$ from Theorem 2 yields $m=\min \left(m, a+2 m\left(\gamma_{m}+1\right)\right)>\frac{\delta}{3} \alpha$. Therefore, the upper bound for $\alpha$ is $\alpha<$ $\min \left(\gamma, \frac{3}{\delta} m\right)$. Concerning the restriction on $\tau$, Theorem 2 with $\tau_{0}=2$ gives $\frac{1}{2}<\frac{1}{\tau}<\frac{1}{\tau^{*}}=\frac{\alpha}{3}+\frac{1}{2}$. This completes the proof.

Remark 10 (The parameter a) We discuss the role of the weight parameter in our Kondratiev spaces: Note that on the one hand we require $a+2 m\left(\gamma_{m}+1\right)>0$ in order to apply the embedding from Theorem 2. Since we assume $a \in[-m, m]$ this is always true. On the other hand it should be expected that the derivatives of the solution $u$ have singularities near the boundary of the polyhedral domain. Thus, looking at the highest derivative of $u(t) \in \mathcal{K}_{2, a+2 m\left(\gamma_{m}+1\right)}^{2 m\left(\gamma_{m}+1\right)}(D)$ we see that we require

$$
\sum_{|\alpha|=2 m\left(\gamma_{m}+1\right)} \int_{D} \rho^{-a p}(x)\left|\partial^{\alpha} u(t, x)\right|^{p} \mathrm{~d} x<\infty,
$$

hence, if $a<0$ the derivatives of the solution $u$ might be unbounded near the boundary of $D$. From this it follows that the range $-m<a<0$ is the most interesting for our considerations.

Remark 11 The above theorem relies on the fact that Problem 1 has a weak solution $u \in H^{m, \gamma_{m}+2 *}\left(D_{T}\right)=H^{\gamma_{m}+1}\left([0, T], \stackrel{\circ}{H}^{m}(D)\right) \cap H^{\gamma_{m}+2}\left([0, T], H^{-m}(D)\right) \hookrightarrow$ $L_{2}\left([0, T], H^{m}(D)\right)$, cf. Theorem 4 . We strongly believe that (in good agreement with the elliptic case) this result can be improved by studying the regularity of Problem 1 in fractional Sobolev spaces $H^{s}(D)$. In this case (assuming that the weak solution of Problem 1 satisfies $u \in L_{2}\left([0, T], H^{s}(D)\right)$ for some $\left.s>0\right)$ under the assumptions of Theorem 8, using Theorem 5 and Theorem 2 (with $k=0$ ), we would obtain

$$
u \in L_{2}\left([0, T], \mathcal{K}_{2, a^{\prime}}^{\alpha}(D)\right) \cap L_{2}\left([0, T], H^{s}(D)\right) \hookrightarrow L_{2}\left([0, T], B_{\tau, \infty}^{\alpha}(D)\right),
$$

where $a^{\prime}=a+2 m\left(\gamma_{m}+1\right) \geq a+2 m$ and again $\frac{1}{2}<\frac{1}{\tau}<\frac{\alpha}{3}+\frac{1}{2}$ but the restriction on $\alpha$ now reads as

$$
\alpha<\frac{3}{\delta} \min \left(s, a^{\prime}\right)
$$

For general Lipschitz domains $D \subset \mathbb{R}^{3}$ we expect that the solution of Problem 1 (for $m=1$ ) is contained in $H^{s}(D)$ for all $s<\frac{3}{2}$ (as in the elliptic case, cf. [32]). This would lead to $\alpha<\frac{9}{2}$ when $\delta=1$. For convex domains it probably even holds that $s=2$ (for the heat equation this was already proven in [45]). First results in this direction can be found in [21]. 
Alternatively, we combine Theorem 6 (Kondratiev regularity B) and Theorem 2. This leads to the following regularity result in Besov spaces.

Theorem 9 (Parabolic Besov regularity B) Let D be a bounded polyhedral domain in $\mathbb{R}^{3}$. Let $\gamma \in \mathbb{N}$ with $\gamma \geq 2 m$. Moreover, let $a \in \mathbb{R}$ with $a \in[-m, m]$. Assume that the right-hand side $f$ of Problem 1 satisfies

(i) $f \in \bigcap_{l=0}^{\infty} H^{l}\left([0, T], L_{2}(D) \cap \mathcal{K}_{2, a}^{\gamma-2 m}(D)\right)$.

(ii) $\partial_{t^{l}} f(x, 0)=0, \quad l \in \mathbb{N}_{0}$.

Furthermore, let Assumption 1 hold for weight parameters $b=a$ and $b^{\prime}=-m$. Then for the weak solution $\bigcap_{l=0}^{\infty} u \in H^{m, l+1 *}\left(D_{T}\right)$ of Problem 1, we have

$$
u \in L_{2}\left([0, T], B_{\tau, \infty}^{\alpha}(D)\right) \text { for all } 0<\alpha<\min \left(\gamma, \frac{3}{\delta} m\right)
$$

where $\frac{1}{2}<\frac{1}{\tau}<\frac{\alpha}{3}+\frac{1}{2}$ and $\delta$ denotes the dimension of the singular set of $D$. In particular, for any $\alpha$ satisfying (5.4) and $\tau$ as above, we have the a priori estimate

$$
\begin{aligned}
& \left\|u\left|L_{2}\left([0, T], B_{\tau, \infty}^{\alpha}(D)\right)\left\|\lesssim \sum_{k=0}^{\gamma-2 m}\right\| \partial_{t^{k}} f\right| L_{2}\left([0, T], \mathcal{K}_{2, a}^{\gamma-2 m}(D)\right)\right\| \\
& +\sum_{k=0}^{(\gamma-2 m)+1}\left\|\partial_{t^{k}} f \mid L_{2}\left(D_{T}\right)\right\| .
\end{aligned}
$$

Proof According to Theorem 6 by our assumptions we know $u \in L_{2}([0, T]$, $\mathcal{K}_{2, a+2 m}^{\gamma}(D)$ ). Together with Theorem 2 (choosing $k=0$ ) we obtain

$$
\begin{aligned}
u \in L_{2} & \left([0, T], \mathcal{K}_{2, a+2 m}^{\gamma}(D)\right) \cap H^{m, 1 *}\left(D_{T}\right) \\
& \hookrightarrow L_{2}\left([0, T], \mathcal{K}_{2, a+2 m}^{\gamma}(D)\right) \cap L_{2}\left([0, T], H^{m}(D)\right) \\
& \hookrightarrow L_{2}\left([0, T], \mathcal{K}_{2, a+2 m}^{\gamma}(D)\right) \cap L_{2}\left([0, T], B_{2, \infty}^{m}(D)\right) \\
& \hookrightarrow L_{2}\left([0, T], \mathcal{K}_{2, a+2 m}^{\alpha}(D) \cap B_{2, \infty}^{m}(D)\right) \hookrightarrow L_{2}\left([0, T], B_{\tau, \infty}^{\alpha}(D)\right),
\end{aligned}
$$

where $\alpha \leq \gamma$ in the second to last line. Moreover, the condition on the parameter ' $a$ ' from Theorem 2 yields $m=\min (m, a+2 m)>\frac{\delta}{3} \alpha$. Therefore, the upper bound for $\alpha$ is $\alpha<\min \left(\gamma, \frac{3}{\delta} m\right)$. Concerning the restriction on $\tau$, Theorem 2 with $\tau_{0}=2$ gives $\frac{1}{2}<\frac{1}{\tau}<\frac{1}{\tau^{*}}=\frac{\alpha}{3}+\frac{1}{2}$. This finishes the proof.

Remark 12 It might not be obvious at first glance that Assumption 1 is satisfied with the parameter restrictions in Theorems 8 and 9. For a discussion on this subject we refer to [22, Rem. 3.8, Ex 4.8], where this matter was discussed in detail and exemplary illustrated for the heat equation. We do not want to repeat the arguments here. 
Fig. 5 Nonlinear solution in $B_{0}$

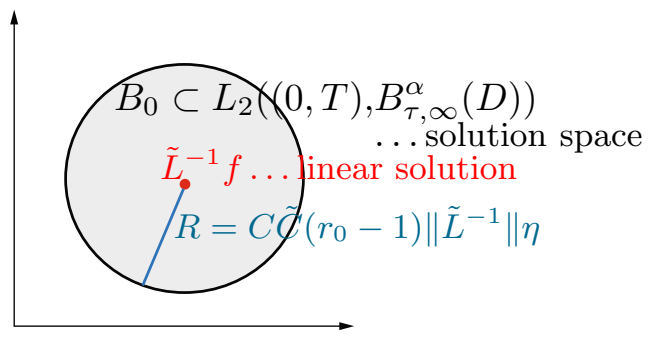

\subsection{Besov Regularity of Problem II}

Concerning the Besov regularity of Problem 2, we proceed in the same way as before for Problem 1: Combining Theorem 7 (Nonlinear Sobolev and Kondratiev regularity) with the embeddings from Theorem 2 we derive the following result.

Theorem 10 (Nonlinear Besov regularity) Let the assumptions of Theorems 7 and 5 be satisfied. In particular, as in Theorem 7 for $\eta:=\|f \mid \widetilde{\mathcal{D}}\|$ and $r_{0}>1$, we choose $\varepsilon>0$ so small that

$$
\eta^{2(M-1)}\left\|\tilde{L}^{-1}\right\|^{2 M-1} \leq \frac{1}{c \varepsilon M}\left(r_{0}-1\right)\left(\frac{1}{r_{0}}\right)^{2 M-1}, \quad \text { if } r_{0}\left\|\tilde{L}^{-1}\right\| \eta>1,
$$

and

$$
\left\|\tilde{L}^{-1}\right\|<\frac{r_{0}-1}{r_{0}}\left(\frac{1}{c \varepsilon M}\right), \quad \text { if } r_{0}\left\|\tilde{L}^{-1}\right\| \eta<1
$$

Then there exists a solution $u$ of Problem 2, which satisfies $u \in B_{0} \subset B$,

$$
B:=L_{2}\left([0, T], B_{\tau, \infty}^{\alpha}(D)\right),
$$

for all $0<\alpha<\min \left(\frac{3}{\delta} m, \gamma\right)$, where $\delta$ denotes the dimension of the singular set of $D$, $\frac{1}{2}<\frac{1}{\tau}<\frac{\alpha}{3}+\frac{1}{2}$, and $B_{0}$ is a small ball around $\tilde{L}^{-1} f$ (the solution of the corresponding linear problem) with radius $R=C \tilde{C}\left(r_{0}-1\right) \eta\left\|\tilde{L}^{-1}\right\|$ (Fig. 5).

Proof This is a consequence of the regularity results in Kondratiev and Sobolev spaces from Theorem 7. To be more precise, Theorem 7 establishes the existence of a fixed point $u$ in

$$
\begin{aligned}
S_{0} \subset S: & =\bigcap_{k=0}^{\gamma_{m}+1} H^{k}\left([0, T], \mathcal{K}_{2, a+2 m\left(\gamma_{m}-(k-1)\right)}^{2 m\left(\gamma_{m}-(k-1)\right)}(D)\right) \cap H^{m, \gamma_{m}+2 *}\left(D_{T}\right) \\
& \hookrightarrow \bigcap_{k=0}^{\gamma_{m}+1} H^{k}\left([0, T], \mathcal{K}_{2, a+2 m\left(\gamma_{m}-(k-1)\right)}^{2 m\left(\gamma_{m}-(k-1)\right)}(D)\right)
\end{aligned}
$$




$$
\begin{gathered}
\cap H^{\gamma_{m}+1}\left([0, T], H^{m}(D)\right) \cap H^{\gamma_{m}+2}\left([0, T], H^{-m}(D)\right) \\
\hookrightarrow L_{2}\left([0, T], \mathcal{K}_{2, a+2 m\left(\gamma_{m}+1\right)}^{2 m\left(\gamma_{m}+1\right)}(K) \cap H^{m}(D)\right)=: \tilde{S} .
\end{gathered}
$$

This together with the embedding results for Besov spaces from Theorem 2 (choosing $k=0$ ) completes the proof, in particular, we calculate for the solution (cf. the proof of Theorem 8)

$$
\begin{aligned}
\| u & -\tilde{L}^{-1} f \mid L_{2}\left([0, T], B_{\tau, \infty}^{\alpha}(D)\right) \| \\
& \leq C\left\|u-\tilde{L}^{-1} f \mid L_{2}\left([0, T], \mathcal{K}_{2, a+2 m\left(\gamma_{m}+1\right)}^{2 m\left(\gamma_{m}+1\right)}(D) \cap H^{m}(D)\right)\right\| \\
& =C\left\|u-\tilde{L}^{-1} f\left|\tilde{S}\|\leq C \tilde{C}\| u-\tilde{L}^{-1} f\right| S\right\| \leq C \tilde{C}\left(r_{0}-1\right) \eta\left\|\tilde{L}^{-1}\right\| .
\end{aligned}
$$

Furthermore, it can be seen from (5.7) that new constants $C$ and $\tilde{C}$ appear when considering the radius $R$ around the linear solution where the problem can be solved compared to Theorem 7 .

Remark 13 A few words concerning the parameters appearing in Theorem 10 (and also Theorem 7) seem to be in order. Usually, the operator norm $\left\|\tilde{L}^{-1}\right\|$ as well as $\varepsilon$ are fixed; but we can change $\eta$ and $r_{0}$ according to our needs. From this we deduce that by choosing $\eta$ small enough the condition (5.6) can always be satisfied. Moreover, it is easy to see that the smaller the nonlinear perturbation $\varepsilon>0$ is, the larger we can choose the radius $R$ of the ball $B_{0}$ where the solution of Problem 2 is unique.

\subsection{Hölder-Besov Regularity of Problem I}

So far we have not exploited the fact that Theorem 5 (Kondratiev regularity A) not only provides regularity properties of the solution $u$ of Problem 1 but also of its partial derivatives $\partial_{t} k$. We use this fact in combination with Theorem 1 in order to obtain some mixed Hölder-Besov regularity results on the whole space-time cylinder $D_{T}$.

For parabolic SPDEs, results in this direction have been obtained in [9]. However, for SPDEs, the time regularity is limited in nature. This is caused by the nonsmooth character of the driving processes. Typically, Hölder regularity $\mathcal{C}^{0, \beta}$ can be obtained, but not more. In contrast to this, it is well known that deterministic parabolic PDEs are smoothing in time. Therefore, in the deterministic case considered here, higher regularity results in time can be obtained compared to the probabilistic setting.

Theorem 11 (Hölder-Besov regularity) Let D be a bounded polyhedral domain in $\mathbb{R}^{3}$. Moreover, let $\gamma \in \mathbb{N}$ with $\gamma \geq 4 m+1$ and put $\gamma_{m}:=\left[\frac{\gamma-1}{2 m}\right]$. Furthermore, let $a \in \mathbb{R}$ with $a \in[-m, m]$. Assume that the right-hand side $f$ of Problem 1 satisfies

(i) $\partial_{t^{k}} f \in L_{2}\left(D_{T}\right) \cap L_{2}\left([0, T], \mathcal{K}_{2, a+2 m\left(\gamma_{m}-k\right)}^{2 m\left(\gamma_{m}-k\right)}(D)\right), \quad k=0, \ldots, \gamma_{m}, \quad \partial_{t^{\prime}+1} f \in$ $L_{2}\left(D_{T}\right)$.

(ii) $\partial_{t^{k}} f(x, 0)=0, \quad k=0,1, \ldots, \gamma_{m}$. 
Let Assumption 1 holdfor weight parameters $b=a+2 m\left(\gamma_{m}-i\right)$, where $i=0, \ldots, \gamma_{m}$ and $b^{\prime}=-m$. Then for the solution $u \in H_{2}^{m, \gamma_{m}+2 *}\left(D_{T}\right)$ of Problem 1 , we have

$$
u \in \mathcal{C}^{\gamma_{m}-2, \frac{1}{2}}\left([0, T], B_{\tau, \infty}^{\eta}(D)\right) \text { for all } 0<\eta<\min \left(\frac{3}{\delta}, 4\right) m,
$$

where $\frac{1}{2}<\frac{1}{\tau}<\frac{\eta}{3}+\frac{1}{2}$ and $\delta$ denotes the dimension of the singular set of $D$. In particular, we have the a priori estimate

$$
\begin{aligned}
& \left\|u\left|\mathcal{C}^{\gamma_{m}-2, \frac{1}{2}}\left([0, T], B_{\tau, \infty}^{\eta}(D)\right)\left\|\lesssim \sum_{k=0}^{\gamma_{m}}\right\| \partial_{t^{k}} f\right| L_{2}\left([0, T], \mathcal{K}_{2, a+2 m\left(\gamma_{m}-k\right)}^{2 m\left(\gamma_{m}-k\right)}(D)\right)\right\| \\
& \quad+\sum_{k=0}^{\gamma_{m}+1}\left\|\partial_{t^{k}} f \mid L_{2}\left(D_{T}\right)\right\|
\end{aligned}
$$

where the constant is independent of $u$ and $f$.

Proof Theorems 5 and 4 show together with Theorems 2 and 1, that under the given assumptions on the initial data $f$, we have for $k \leq \gamma_{m}-2$,

$$
\begin{aligned}
u & \in H^{k+1}\left([0, T], \mathcal{K}_{2, a+2 m\left(\gamma_{m}-k\right)}^{2 m\left(\gamma_{m}-k\right)}(D)\right) \cap H^{\gamma_{m}+1}\left([0, T], H^{m}(D)\right) \\
& \hookrightarrow H^{k+1}\left([0, T], \mathcal{K}_{2, a+2 m\left(\gamma_{m}-k\right)}^{2 m\left(\gamma_{m}-k\right)}(K) \cap H^{m}(D)\right) \\
& \hookrightarrow \mathcal{C}^{k, \frac{1}{2}}\left([0, T], \mathcal{K}_{2, a+2 m\left(\gamma_{m}-k\right)}^{2 m\left(\gamma_{m}-k\right)}(D) \cap H^{m}(D)\right) \\
\hookrightarrow & \mathcal{C}^{k, \frac{1}{2}}\left([0, T], \mathcal{K}_{2, a+2 m\left(\gamma_{m}-k\right)}^{\eta}(D) \cap H^{m}(D)\right) \hookrightarrow \mathcal{C}^{k, \frac{1}{2}}\left([0, T], B_{\tau, \infty}^{\eta}(D)\right),
\end{aligned}
$$

where in the third step we require $\eta \leq 2 m\left(\gamma_{m}-k\right)$ and by Theorem 2 we get the additional restriction $m=\min \left(m, a+2 m\left(\gamma_{m}-k\right)\right) \geq \frac{\delta}{3} \eta$, i.e., $\eta<\frac{3}{\delta} m$. Therefore, the upper bound on $\eta$ reads as $\eta<\min \left(\frac{3}{\delta} m, 2 m\left(\gamma_{m}-k\right)\right)$ since $k \leq \gamma_{m}-2$, which for $k=\gamma_{m}-2$ yields $\eta<\min \left(\frac{3}{\delta}, 4\right) m$.

Remark 14 (i) For $\gamma \geq 2 m+1$ and $k=\gamma_{m}-1$ we have $\eta \leq \min \left(\frac{3}{\delta}, 2\right) m$ in the theorem above. For $\gamma \geq 2 m$ and $k=\gamma_{m}$ we get $\eta=0$.

(ii) From the proof of Theorem 11 above it can be seen that the solution satisfies $u \in \mathcal{C}^{k, \frac{1}{2}}\left([0, T], \mathcal{K}_{2, a+2 m\left(\gamma_{m}-k\right)}^{2 m\left(\gamma_{m}-k\right)}(D)\right)$, implying that for high regularity in time, which is displayed by the parameter $k$, we have less spatial regularity in terms of $2 m\left(\gamma_{m}-k\right)$.

Funding Open Access funding enabled and organized by Projekt DEAL.

Open Access This article is licensed under a Creative Commons Attribution 4.0 International License, which permits use, sharing, adaptation, distribution and reproduction in any medium or format, as long as you give appropriate credit to the original author(s) and the source, provide a link to the Creative Commons licence, and indicate if changes were made. The images or other third party material in this article are included 
in the article's Creative Commons licence, unless indicated otherwise in a credit line to the material. If material is not included in the article's Creative Commons licence and your intended use is not permitted by statutory regulation or exceeds the permitted use, you will need to obtain permission directly from the copyright holder. To view a copy of this licence, visit http://creativecommons.org/licenses/by/4.0/.

\section{A Appendix}

\section{A.1 Preliminaries}

We collect some notation used throughout the paper. As usual, we denote by $\mathbb{N}$ the set of all natural numbers, $\mathbb{N}_{0}=\mathbb{N} \cup\{0\}$, and $\mathbb{R}^{d}, d \in \mathbb{N}$, the $d$-dimensional real Euclidean space with $|x|$, for $x \in \mathbb{R}^{d}$, denoting the Euclidean norm of $x$. By $\mathbb{Z}^{d}$ we denote the lattice of all points in $\mathbb{R}^{d}$ with integer components. For $a \in \mathbb{R}$, let $[a]$ denote its integer part.

Moreover, $c$ stands for a generic positive constant which is independent of the main parameters, but its value may change from line to line. The expression $A \lesssim B$ means that $A \leq c B$. If $A \lesssim B$ and $B \lesssim A$, then we write $A \sim B$.

Given two quasi-Banach spaces $X$ and $Y$, we write $X \hookrightarrow Y$ if $X \subset Y$ and the natural embedding is bounded. By supp $f$ we denote the support of the function $f$. For a domain $\Omega \subset \mathbb{R}^{d}$ and $r \in \mathbb{N} \cup\{\infty\}$ we write $C^{r}(\Omega)$ for the space of all realvalued $r$-times continuously differentiable functions, whereas $C(\Omega)$ is the space of bounded uniformly continuous functions, and $\mathcal{D}(\Omega)$ for the set of test functions, i.e., the collection of all infinitely differentiable functions with compact support contained in $\Omega$. Moreover, $L_{\text {loc }}^{1}(\Omega)$ denotes the space of locally integrable functions on $\Omega$.

For a multi-index $\alpha=\left(\alpha_{1}, \ldots, \alpha_{d}\right) \in \mathbb{N}_{0}^{d}$ with $|\alpha|:=\alpha_{1}+\cdots+\alpha_{d}=r, r \in \mathbb{N}_{0}$, and an $r$-times differentiable function $u: \Omega \rightarrow \mathbb{R}$, we write

$$
D^{(\alpha)} u=\frac{\partial^{|\alpha|}}{\partial x_{1}^{\alpha_{1}} \ldots \partial x_{d}^{\alpha_{d}}} u
$$

for the corresponding classical partial derivative as well as $u^{(k)}:=D^{(k)} u$ in the onedimensional case. Hence, the space $C^{r}(\Omega)$ is normed by

$$
\left\|u\left|C^{r}(\Omega) \|:=\max _{|\alpha| \leq r} \sup _{x \in \Omega}\right| D^{(\alpha)} u(x) \mid<\infty\right.
$$

Moreover, $\mathcal{S}\left(\mathbb{R}^{d}\right)$ denotes the Schwartz space of rapidly decreasing functions. The set of distributions on $\Omega$ will be denoted by $\mathcal{D}^{\prime}(\Omega)$, whereas $\mathcal{S}^{\prime}\left(\mathbb{R}^{d}\right)$ denotes the set of tempered distributions on $\mathbb{R}^{d}$. The terms distribution and generalized function will be used synonymously. For the application of a distribution $u \in \mathcal{D}^{\prime}(\Omega)$ to a test function $\varphi \in \mathcal{D}(\Omega)$ we write $(u, \varphi)$. The same notation will be used if $u \in \mathcal{S}^{\prime}\left(\mathbb{R}^{d}\right)$ and $\varphi \in \mathcal{S}\left(\mathbb{R}^{d}\right)$ (and also for the inner product in $L_{2}(\Omega)$ ). For $u \in \mathcal{D}^{\prime}(\Omega)$ and a multiindex $\alpha=\left(\alpha_{1}, \ldots, \alpha_{d}\right) \in \mathbb{N}_{0}^{d}$, we write $D^{\alpha} u$ for the $\alpha$-th generalized or distributional derivative of $u$ with respect to $x=\left(x_{1}, \ldots, x_{d}\right) \in \Omega$, i.e., $D^{\alpha} u$ is a distribution on $\Omega$, 
uniquely determined by the formula

$$
\left(D^{\alpha} u, \varphi\right):=(-1)^{|\alpha|}\left(u, D^{(\alpha)} \varphi\right), \quad \varphi \in \mathcal{D}(\Omega) .
$$

In particular, if $u \in L_{\text {loc }}^{1}(\Omega)$ and there exists a function $v \in L_{\text {loc }}^{1}(\Omega)$ such that

$$
\int_{\Omega} v(x) \varphi(x) \mathrm{d} x=(-1)^{|\alpha|} \int_{\Omega} u(x) D^{(\alpha)} \varphi(x) \mathrm{d} x \quad \text { for all } \quad \varphi \in \mathcal{D}(\Omega),
$$

we say that $v$ is the $\alpha$-th weak derivative of $u$ and write $D^{\alpha} u=v$. We also use the notation $\frac{\partial^{k}}{\partial x_{j}^{k}} u:=D^{\beta} u$ as well as $\partial_{x_{j}^{k}}:=D^{\beta} u$, for some multi-index $\beta=(0, \ldots, k, \ldots, 0)$ with $\beta_{j}=k, k \in \mathbb{N}$. Furthermore, for $m \in \mathbb{N}_{0}$, we write $D^{m} u$ for any (generalized) $m$-th order derivative of $u$, where $D^{0} u:=u$ and $D u:=D^{1} u$. Sometimes we shall use subscripts such as $D_{x}^{m}$ or $D_{t}^{m}$ to emphasize that we only take derivatives with respect to $x=\left(x_{1}, \ldots, x_{d}\right) \in \Omega$ or $t \in \mathbb{R}$.

\section{Besov Spaces}

Due to the different contexts Besov spaces arose from they can be defined/characterized in several ways, e.g., via higher order differences, the Fourier-analytic approach or decompositions with suitable building blocks, cf. $[43,44]$ and the references therein. Under certain restrictions on the parameters these different approaches might even coincide. Throughout this paper, we rely on the characterization of Besov spaces via wavelet decompositions and refer in this context to [11,38]. Let us briefly recall the concept: Wavelets are specific orthonormal bases for $L_{2}(\mathbb{R})$ that are obtained by dilating, translating, and scaling one fixed function, the so-called mother wavelet $\psi$. The mother wavelet is usually constructed by means of a so-called multiresolution analysis, that is, a sequence $\left\{V_{j}\right\}_{j \in \mathbb{Z}}$ of shift-invariant, closed subspaces of $L_{2}(\mathbb{R})$ whose union is dense in $L_{2}$ while their intersection is trivial. Moreover, all the spaces are related via dyadic dilation, and the space $V_{0}$ is spanned by the translates of one fixed function $\phi$, called the generator. In her fundamental work [24,25] I. Daubechies has shown that there exist families of compactly supported wavelets. By taking tensor products, a compactly supported orthonormal basis for $L_{2}\left(\mathbb{R}^{d}\right)$ can be constructed.

Let $\phi$ be a father wavelet of tensor product type on $\mathbb{R}^{d}$ and let $\Psi^{\prime}=\left\{\psi_{i}: i=\right.$ $\left.1, \ldots, 2^{d}-1\right\}$ be the set containing the corresponding multivariate mother wavelets such that, for a given $r \in \mathbb{N}$ and some $N>0$ the following localization, smoothness, and vanishing moment conditions hold. For all $\psi \in \Psi^{\prime}$,

$$
\begin{aligned}
\operatorname{supp} \phi, \operatorname{supp} \psi & \subset[-N, N]^{d}, \\
\phi, \psi & \in C^{r}\left(\mathbb{R}^{d}\right), \\
\int_{\mathbb{R}^{d}} x^{\alpha} \psi(x) \mathrm{d} x & =0 \quad \text { for all } \alpha \in \mathbb{N}_{0}^{d} \text { with }|\alpha| \leq r .
\end{aligned}
$$


We refer again to $[24,25]$ for a detailed discussion. The set of all dyadic cubes in $\mathbb{R}^{d}$ with measure at most 1 is denoted by

$$
\mathcal{D}^{+}:=\left\{I \subset \mathbb{R}^{d}: I=2^{-j}\left([0,1]^{d}+k\right), j \in \mathbb{N}_{0}, k \in \mathbb{Z}^{d}\right\}
$$

and we set $\mathcal{D}_{j}:=\left\{I \in \mathcal{D}^{+}:|I|=2^{-j d}\right\}$. For the dyadic shifts and dilations of the father wavelet and the corresponding wavelets we use the abbreviations

$\phi_{k}(x):=\phi(x-k), \quad \psi_{I}(x):=2^{j d / 2} \psi\left(2^{j} x-k\right) \quad$ for $\quad j \in \mathbb{N}_{0}, k \in \mathbb{Z}^{d}, \psi \in\left(\mathbb{H}^{\prime} .4\right)$

It follows that

$$
\left\{\phi_{k}, \psi_{I}: k \in \mathbb{Z}^{d}, I \in \mathcal{D}^{+}, \psi \in \Psi^{\prime}\right\}
$$

is an orthonormal basis in $L_{2}\left(\mathbb{R}^{d}\right)$. Denote by $Q(I)$ some dyadic cube (of minimal size) such that supp $\psi_{I} \subset Q(I)$ for every $\psi \in \Psi^{\prime}$. Then, we clearly have $Q(I)=$ $2^{-j} k+2^{-j} Q$ for some dyadic cube $Q$. Put $\Lambda^{\prime}=\mathcal{D}^{+} \times \Psi^{\prime}$. Then, every function $f \in L_{2}\left(\mathbb{R}^{d}\right)$ can be written as

$$
f=\sum_{k \in \mathbb{Z}^{d}}\left\langle f, \phi_{k}\right\rangle \phi_{k}+\sum_{(I, \psi) \in \Lambda^{\prime}}\left\langle f, \psi_{I}\right\rangle \psi_{I}
$$

It will be convenient to include $\phi$ into the set $\Psi^{\prime}$. We use the notation $\phi_{I}:=0$ for $|I|<1, \phi_{I}=\phi(\cdot-k)$ for $I=k+[0,1]^{d}$, and can simply write

$$
f=\sum_{(I, \psi) \in \Lambda}\left\langle f, \psi_{I}\right\rangle \psi_{I}, \quad \Lambda=\mathcal{D}^{+} \times \Psi, \quad \Psi=\Psi^{\prime} \cup\{\phi\} .
$$

We describe Besov spaces on $\mathbb{R}^{d}$ by decay properties of the wavelet coefficients, if the parameters fulfill certain conditions.

Theorem A.1 (Wavelet decomposition of Besov spaces) Let $0<p, q<\infty$, and $s>\max \{0, d(1 / p-1)\}$. Choose $r \in \mathbb{N}$ such that $r>s$ and construct a wavelet Riesz basis as described above. Then a function $f \in L_{p}\left(\mathbb{R}^{d}\right)$ belongs to the Besov space $B_{p, q}^{s}\left(\mathbb{R}^{d}\right)$ if, and only if,

$$
f=\sum_{k \in \mathbb{Z}^{d}}\left\langle f, \phi_{k}\right\rangle \phi_{k}+\sum_{(I, \psi) \in \Lambda^{\prime}}\left\langle f, \psi_{I}\right\rangle \psi_{I}
$$

(convergence in $\mathcal{S}^{\prime}\left(\mathbb{R}^{d}\right)$ ) with

$$
\left\|f \mid B_{p, q}^{s}\left(\mathbb{R}^{d}\right)\right\| \sim\left(\sum_{k \in \mathbb{Z}^{d}}\left|\left\langle f, \phi_{k}\right\rangle\right|^{p}\right)^{1 / p}+
$$




$$
\left(\sum_{j=0}^{\infty} 2^{j\left(s+d\left(\frac{1}{2}-\frac{1}{p}\right)\right) q}\left(\sum_{(I, \psi) \in \mathcal{D}_{j} \times \Psi^{\prime}}\left|\left\langle f, \psi_{I}\right\rangle\right|^{p}\right)^{q / p}\right)^{1 / q}<\infty
$$

Remark $A .1$ In particular, for our adaptivity scale (1.2), i.e., $B_{\tau, \tau}^{s}\left(\mathbb{R}^{d}\right)$ with $s=$ $d\left(\frac{1}{\tau}-\frac{1}{p}\right)$, we see that the quasi-norm (A.6) becomes

$\left\|f \mid B_{\tau, \tau}^{s}\left(\mathbb{R}^{d}\right)\right\| \sim\left(\sum_{k \in \mathbb{Z}^{d}}\left|\left\langle f, \phi_{k}\right\rangle\right|^{\tau}\right)^{1 / \tau}+\left(\sum_{j=0}^{\infty} 2^{j d\left(\frac{1}{2}-\frac{1}{p}\right) \tau} \sum_{(I, \psi) \in \mathcal{D}_{j} \times \Psi^{\prime}}\left|\left\langle f, \psi_{I}\right\rangle\right|^{\tau}\right)^{1 / \tau}$.

Corresponding function spaces on domains $\mathcal{O} \subset \mathbb{R}^{d}$ can be introduced via restriction, i.e.,

$$
\begin{aligned}
B_{p, q}^{s}(\mathcal{O}) & =\left\{f \in \mathcal{D}^{\prime}(\mathcal{O}): \exists g \in B_{p, q}^{s}\left(\mathbb{R}^{d}\right),\left.g\right|_{\mathcal{O}}=f\right\}, \\
\left\|f \mid B_{p, q}^{s}(\mathcal{O})\right\| & =\inf _{\left.g\right|_{\mathcal{O}=f}}\left\|f \mid B_{p, q}^{s}\left(\mathbb{R}^{d}\right)\right\| .
\end{aligned}
$$

Alternative (different or equivalent) versions of this definition can be found, depending on possible additional properties of the distributions $g$ (most often their support). We refer to [44] for details and references.

Remark A.2 We remark that the Besov (and Kondratiev) spaces we are working with are defined in the setting of distributions, i.e., as subsets of $\mathcal{D}^{\prime}(\mathcal{O})$, and therefore may contain 'functions' which take complex values. However, when considering the fundamental parabolic problems, we restrict ourselves to the real-valued setting: We assume the coefficients of the differential operator $L$ to be real-valued as well as the right-hand side $f$, therefore, the solutions are real-valued as well.

\section{References}

1. Agmon, S., Douglis, A., Nierenberg, L.: Estimates near the boundary for solutions of elliptic partial differential equations satisfying general boundary conditions I. Commun. Pure Appl. Math. 12, 623727 (1959)

2. Aimar, H., Gómez, I., Iaffei, B.: Parabolic mean values and maximal estimates for gradients of temperatures. J. Funct. Anal. 255, 1939-1956 (2008)

3. Aimar, H., Gómez, I., Iaffei, B.: On Besov regularity of temperatures. J. Fourier Anal. Appl. 16, 1007-1020 (2010)

4. Aimar, H., Gómez, I.: Parabolic Besov regularity for the heat equation. Constr. Approx. 36, 145-159 (2012)

5. Anh, N.T., Hung, N.M.: Regularity of solutions of initial-boundary value problems for parabolic equations in domains with conical points. J. Differ. Equ. 245(7), 1801-1818 (2008)

6. Anh, N.T., Loi, D.V., Luong, V.T.: $L_{p}$-regularity for the Cauchy-Dirichlet problem for parabolic equations in convex polyhedral domains. Acta Math. Vietnam. 41(4), 731-742 (2016) 
7. Babuska, I., Guo, B.: Regularity of the solutions for elliptic problems on nonsmooth domains in $\mathbb{R}^{3}$, Part I: countably normed spaces on polyhedral domains. Proc. R. Soc. Edinburgh Sect. A 127, 77-126 (1997)

8. Bacuta, C., Mazzucato, A., Nistor, V., Zikatanov, L.: Interface and mixed boundary value problems on $n$-dimensional polyhedral domains. Doc. Math. 15, 687-745 (2010)

9. Cioica, P., Kim, K.-H., Lee, K., Lindner, F.: On the $L_{q}\left(L_{p}\right)$-regularity and Besov smoothness of stochastic parabolic equations on bounded Lipschitz domains. Electron. J. Probab. 18(82), 1-41 (2013)

10. Cioica-Licht, P., Weimar, M.: On the limit regularity in Sobolev and Besov scales related to approximation theory. J. Fourier Anal. Appl. 26(1), 10 (2020)

11. Cohen, A.: Numerical Analysis of Wavelet Methods. Studies in Mathematics and Its Applications, vol. 32, 1st edn. Elsevier, Amsterdam (2003)

12. Costabel, M.: On the limit Sobolev regularity for Dirichlet and Neumann problems on Lipschitz domains. Math. Nachr. 292, 2165-2173 (2019)

13. Dahlke, S.: Besov regularity for elliptic boundary value problems with variable coefficients. Manuscripta Math. 95, 59-77 (1998)

14. Dahlke, S.: Besov regularity for interface problems. Z. Angew. Math. Mech. 79(6), 383-388 (1999)

15. Dahlke, S.: Besov regularity for elliptic boundary value problems on polygonal domains. Appl. Math. Lett. 12(6), 31-38 (1999)

16. Dahlke, S.: Besov regularity of edge singularities for the Poisson equation in polyhedral domains. Num. Linear Algebra Appl. 9(6-7), 457-466 (2002)

17. Dahlke, S., Dahmen, W., DeVore, R.: Nonlinear approximation and adaptive techniques for solving elliptic operator equations. In: Dahmen, W., Kurdila, A.J., Oswald, P. (eds.) Multiscale Wavelet Methods for Partial Differential Equations. Wavelet Analysis and Applications, vol. 6, pp. 237-283. Academic Press, San Diego (1997)

18. Dahlke, S., DeVore, R.: Besov regularity for elliptic boundary value problems. Commun. Partial Differ. Equ. 22(1-2), 1-16 (1997)

19. Dahlke, S., Diening, L., Hartmann, C., Scharf, B., Weimar, M.: Besov regularity of solutions to the p-Poisson equation. Nonlinear Anal. 130(298-329), 2016 (2016)

20. Dahlke, S., Hansen, M., Schneider, C., Sickel, W.: Properties of Kondratiev spaces. Reihe Philipps University Marburg, Bericht Mathematik Nr. 2018-06. (2018). arXiv:1911.01962

21. Dahlke, S., Schneider, C.: Describing the singular behaviour of parabolic equations on cones in fractional Sobolev spaces. Int. J. Geomath. 9(2), 293-315 (2018)

22. Dahlke, S., Schneider, C.: Besov regularity of parabolic and hyperbolic PDEs. Anal. Appl. 17(2), 235-291 (2019)

23. Dahlke, S., Novak, E., Sickel, W.: Optimal approximation of elliptic problems by linear and nonlinear mappings. II. J. Complex. 22, 549-603 (2006)

24. Daubechies, I.: Orthonormal bases of compactly supported wavelets. Commun. Pure Appl. Math. 41(7), 909-996 (1998)

25. Daubechies, I.: Ten lectures on wavelets. In: CBMS-NSF Regional Conference Series in Applied Mathematics, vol. 61. SIAM, Philladelphia (1992)

26. Evans, L.C.: Partial differential equations. Graduate Studies in Mathematics, vol. 19, 2nd edn. American Mathematical Society, Providence, RI (2010)

27. Gaspoz, F.D., Morin, P.: Convergence rates for adaptive finite elements. IMA J. Numer. Anal. 29(4), 917-936 (2009)

28. Grisvard, P.: Singularities in Boundary Value Problems. Recherches en Mathématiques Appliquées, vol. 22. Masson, Paris; Springer-Verlag, Berlin (1992)

29. Grisvard, P.: Elliptic problems in nonsmooth domains. Reprint of the 1985 original. Classics in Applied Mathematics, vol. 69. SIAM, Philadelphia (2011)

30. Hansen, M.: Nonlinear approximation rates and Besov regularity for elliptic PDEs on polyhedral domains. Found. Comput. Math. 15, 561-589 (2015)

31. Hartmann, C., Weimar, M.: Besov regularity of solutions to the $p$-Poisson equation in the vicinity of a vertex of a polygonal domain. Results Math. 73(41), 1-28 (2018)

32. Jerison, D., Kenig, C.E.: The inhomogeneous Dirichlet problem in Lipschitz domains. J. Funct. Anal. 130, 161-219 (1995)

33. Kozlov, V.A., Mazya, V.G., Rossman, J.: Spectral Problems Associated with Corner Singularities of Solutions to Elliptic Equations. Mathematical Surveys and Monographs, vol. 85. American Mathematical Society, Providence, RI (2001) 
34. Lang, J.: Adaptive Multilevel Solution of Nonlinear Parabolic PDE Systems. Lecture Notes in Computational Science and Engineering; Theory, Algorithm, and Applications, vol. 16. Springer-Verlag, Berlin (2001)

35. Luong, V.T., Loi, D.V.: The first initial-boundary value problem for parabolic equations in a cone with edges. Vestn. St.-Petersbg. Univ. Ser. 1. Mat. Mekh. Asron. 2, 60(3), 394-404 (2015)

36. Maz'ya, V., Rossmann, J.: Elliptic Equations in Polyhedral Domains. Mathematical Surveys and Monographs, vol. 162. American Mathematical Society, New York (2010)

37. Mazzucato, A., Nistor, V.: Well posedness and regularity for the elasticity equation with mixed boundary conditions on polyhedral domains and domains with cracks. Arch. Ration. Mech. Anal. 195, 25-73 (2010)

38. Meyer, Y.: Wavelets and Operators. Cambridge Studies in Advances Mathematics, vol. 37. Cambridge University Press, Cambridge (1992)

39. Runst, T., Sickel, W.: Sobolev spaces of fractional order, Nemytskij operators, and nonlinear partial differential equations. De Gruyter Series in Nonlinear Analysis and Applications (1996)

40. Schneider, C.: Besov regularity of partial differential equations, and traces in function spaces. PhilippsUniversität Marburg, Habilitationsschrift (2020)

41. Stevenson, R., Schwab, C.: Space-time adaptive wavelet methods for parabolic evolution problems. Math. Comput. 78, 1293-1318 (2009)

42. Thomée, V.: Galerkin Finite Element Methods for Parabolic Problems. Springer Series in Computational Mathematics, vol. 25, 2nd edn. Springer-Verlag, Berlin (2006)

43. Triebel, H.: Theory of Function Spaces Monographs in Mathematics, vol. 78. Birkhäuser Verlag, Basel (1983)

44. Triebel, H.: Function Spaces and Wavelets on Domains. EMS Tracts on Mathematics, vol. 7. EMS Publishing House, Zürich (2008)

45. Wood, I.: Maximal $L^{p}$-regularity for the Laplacian on Lipschitz domains. Math. Z. 255(4), 855-875 (2007)

Publisher's Note Springer Nature remains neutral with regard to jurisdictional claims in published maps and institutional affiliations. 